\title{
Les communautés montagnardes et la justice dans les Alpes nord-occidentales à la fin du Moyen Âge
} Chamonix, Abondance et les régions voisines, $\mathrm{XIV}^{\mathrm{e}}-\mathrm{XV}^{\mathrm{e}}$ siècles

\section{Nicolas Carrier}

\section{OpenEdition}

\section{Journals}

Édition électronique

URL : https://journals.openedition.org/crm/1573

DOI : 10.4000/crm.1573

ISSN : 1955-2424

\section{Éditeur}

Honoré Champion

Édition imprimée

Date de publication : 15 mars 2003

Pagination : 89-118

ISSN : 1272-9752

\section{Référence électronique}

Nicolas Carrier, «Les communautés montagnardes et la justice dans les Alpes nord-occidentales à la fin du Moyen Âge », Cahiers de recherches médiévales [En ligne], $10 \mid$ 2003, mis en ligne le 08 octobre 2007, consulté le 15 décembre 2022. URL : http://journals.openedition.org/crm/1573 ; DOI : https:// doi.org/10.4000/crm.1573

Ce document a été généré automatiquement le 15 décembre 2022.

Tous droits réservés 


\section{Les communautés montagnardes et la justice dans les Alpes nord- occidentales à la fin du Moyen Âge}

Chamonix, Abondance et les régions voisines, $\mathrm{XIV}^{\mathrm{e}}-\mathrm{XV}^{\mathrm{e}}$ siècles

\section{Nicolas Carrier}

1 Libres montagnards. Telle est l'image qu'une tradition historiographique déjà longue a solidement installée, à partir de quelques exemples de vigoureuses communautés alpines ou pyrénéennes, comme les "escartons" du Briançonnais, les Waldstetten helvétiques, les communautés pastorales de la Vésubie ou celles de l'Andorre ${ }^{1}$.

2 En Savoie aussi, la tradition historiographique régionale veut que les communautés montagnardes soient spécialement autonomes et actives. Deux arguments sont habituellement invoqués: l'importance des associations de copropriétaires ou «consortages», en lien avec l'activité pastorale ${ }^{2}$; les prérogatives judiciaires dont disposent certaines communautés de montagne. Ce dernier thème historiographique est fort ancien, puisqu'à notre connaissance, l'origine s'en trouve dans une communication faite par André Perrin au congrès des Sociétés savantes de Savoie de 1879. Préparant alors une édition du chartrier du prieuré bénédictin de Chamonix et une Histoire de la vallée et du prieuré de Chamonix ${ }^{3}$, ce notable et érudit local remarquait avec étonnement qu'à la fin du Moyen Âge, dans cette vallée du massif du Mont-Blanc, "l'exercice de la justice criminelle était réservé aux syndics ou aux bons-hommes élus par leurs pairs à l'exclusion des prieurs, maîtres du sol, et de leurs juges ». Il citait en outre deux procès montrant que les paysans dépendants de l'abbaye chablaisienne d'Abondance exerçaient eux aussi la haute justice. Il n'hésitait d'ailleurs pas à conclure: «cette importante prérogative, qui remontait aux libertés germaines, fut maintenue intacte pendant toute la durée du Moyen Âge dans la plupart des hautes vallées de la Savoie et du Valais $»^{4}$. Pour cet auteur, l'exercice de la haute justice par les communautés paysannes était un privilège à la fois fort ancien, largement partagé dans les hautes vallées des Alpes nord-occidentales, et lié au farouche esprit d'indépendance des montagnards, qui seuls auraient su conserver les « libertés germaines » menacées 
par la «féodalité». Trois affirmations sur lesquelles les historiens régionaux l'ont d'abord suivi de confiance ${ }^{5}$, puis de plus en plus prudemment, à mesure qu'ils évaluaient mieux l'importance de la mutation féodale et qu'ils devaient reconnaitre que les archives savoyardes demeuraient peu loquaces sur la question. Reste que le paradigme d'une liberté particulière aux communautés de montagne était si communément reçu que ces deux exemples toujours repris de Perrin passaient pour les arbres dissimulant une forêt de libertés restant à découvrir.

Il se trouve que certaines des études les plus récentes ont amené à remettre en cause le paradigme. Henri Falque-Vert a montré par exemple que dans la seconde moitié du XIII ${ }^{e}$ siècle les paysans du Haut-Dauphiné sont très majoritairement des serfs, à ce titre mainmortables et soumis à une taille le plus souvent arbitraire ${ }^{6}$. Encore sont-ils libérés de la servitude par la charte de franchises de 1343, qui marque en outre leur adhésion à la confédération des vallées briançonnaises. Ce n'est pas le cas des montagnards du Faucigny qui, eux aussi, sont serfs dans leur grande majorité et le resteront jusqu'aux affranchissements du XVIII siècle. Nos recherches sur les communautés faucignerandes nous ont d'ailleurs amené à conclure que l'omniprésence des consortages ne débouchait nullement, à la différence par exemple de ce qu'on constate en Vésubie, sur une autonomie politique ${ }^{7}$. Certes, la communauté de Chamonix, qui dispose de franchises écrites depuis 1292, qui fait preuve régulièrement de mauvaise humeur à l'égard de ses prieurs, qui obtient progressivement un adoucissement de la mainmorte, fait un peu moins pâle figure que les communautés voisines, mais c'est surtout en regard de l'inexistence politique de ces dernières.

4 À la lumière d'un contexte social et seigneurial mieux connu aujourd'hui, nous avons souhaité reprendre sur pièces le dossier des privilèges judiciaires des communautés de montagne des Alpes nord-occidentales. Nous partirons des cas d'Abondance et de Chamonix, qui sont à la fois les mieux documentés et les plus originaux. En effet, comme André Perrin l'avait remarqué en son temps, la connaissance des causae criminales corporales ${ }^{8}$, c'est-à-dire des procès pénaux où l'accusé encourt un châtiment corporel, y revenait à la fin du Moyen Âge aux paysans du lieu. En d'autres termes, ceux-ci exerçaient la haute justice, bien qu'ils fussent sous la domination banale d'un seigneur ecclésiastique lui-aussi réputé haut-justicier. Dans un second temps, nous aurons soin d'éclairer ces cas en les replaçant dans le cadre plus large des libertés obtenues par les communautés de la Savoie, du Dauphiné et de l'actuelle Suisse romande, sans craindre d'ailleurs d'étendre nos comparaisons aux communautés urbaines, qu'il n'y a pas lieu d'exclure du point de vue qui nous occupe'.

5 Par cette étude approfondie de deux cas particuliers et par ce tableau général dressé dans un cadre plus large, nous tâcherons d'apporter un éclairage montagnard à la problématique des paysans dans leurs communautés, nous demandant particulièrement s'il existe vraiment des libertés propres aux communautés de montagne.

Deux communautés de montagne défendent et étendent leurs privilèges judiciaires

6 Séparés de quelques dizaines de kilomètres à vol d'oiseau, le prieuré de Chamonix et l'abbaye d'Abondance sont deux monastères de montagne. Occupant les hautes vallées des deux rivières de l'Arve et de la Dranse d'Abondance, ils n'ont pas la même importance dans la géographie ecclésiastique des Alpes du nord.

7 Le prieuré bénédictin de Chamonix est une fondation de l'abbaye piémontaise de La Cluse. Il s'est installé dans la vallée séparant les massifs du Mont-Blanc et des Aiguilles 
rouges, concédée entre 1089 et 1099 par le comte de Genève Aymon Ir ${ }^{\text {er }}$. N'ayant jamais dépassé la dizaine de moines, il n'a pas essaimé. Son temporel ne s'est guère étendu audelà de la donation initiale. À la fin du XIII ${ }^{\mathrm{e}}$ siècle, le mandement de Chamonix correspondait aux paroisses de Chamonix et de Vallorcine et à une partie de celle de Notre-Dame du Lac ${ }^{11}$.

Fondation de l'abbaye Saint-Maurice d'Agaune, un prieuré de chanoines augustins existe à Abondance au tournant des $\mathrm{XI}^{\mathrm{e}}$-XII ${ }^{\mathrm{e}}$ siècles. Il devient abbaye avant 1140 et essaimera par la suite, notamment à Sixt et à Entremont. En 1108, son temporel est séparé de celui d'Agaune. Il correspond alors à la haute vallée de la Dranse d'Abondance, rivière qui prend sa source dans le massif du Chablais ${ }^{12}$. Ce temporel initial du monastère est ce qu'on a accoutumé d'appeler la vallée d'Abondance. À une date inconnue, les chanoines l'ont divisée en deux paroisses, celles d'Abondance et de la Chapelle des Frasses, qui correspondent aux actuelles communes d'Abondance, de la Chapelle d'Abondance et de Châtel ${ }^{13}$. Le monastère fut favorisé depuis le XII ${ }^{\mathrm{e}}$ siècle de nombreuses donations, qui lui permirent d'acquérir des biens et des droits dans une grande partie du Chablais, de la vallée d'Abondance aux rives du Léman, avec des droits de haute justice dans plusieurs localités ${ }^{14}$.

Chamonix

9 L'universitas de Chamonix ${ }^{15}$ est mentionnée pour la première fois en 1264. Elle regroupe les habitants de la paroisse de Chamonix et, dans un conflit de bornage qui l'oppose à une communauté voisine, se trouve alors représentée par le prieur du lieu. En 1289 apparaît une communitas de Chamonix, au sens de commune jurée par les Chamoniards cette fois révoltés contre leur prieur, avec l'aide du comte de Genève. L'affaire se clôt en 1292 par un arbitrage amiable rendu par des personnes choisies d'un commun accord par les deux parties. C'est la manière la plus commune de résoudre les conflits dans les communautés qui nous occupent. En l'occurrence, cette sentence arbitrale tranche les différents points de conflit qui opposent le prieur et ses hommes et tient lieu de première charte de franchises de Chamonix. Elle ne concerne que la seule paroisse de Chamonix, celles de Vallorcine et de Notre-Dame du Lac en étant explicitement exclues. Une nouvelle sentence arbitrale est rendue en 1330 ; elle vaut cette fois pour tous les dépendants du prieuré, dans quelqu'une des trois paroisses qu'ils habitent, en tant qu'ils sont collectivement possesseurs des communaux. D'autres arbitrages interviennent en 1368, 1386, 1421 et 1441, pour trancher différents points controversés. En 1493 enfin, une transaction amiable, sans intervention d'arbitres, met fin à un long procès.

Ce sont ces différents textes que les habitants de la vallée de Chamonix regardent comme contenant leurs franchises. Dans l'ensemble, ils marquent toujours des conquêtes des Chamoniards, notamment en ce qui concerne la mainmorte, dont le régime est de plus en plus adouci. Tous regardent également peu ou prou les prérogatives judiciaires de la communauté. Ce n'est qu'en 1441 que les syndics de Chamonix, qu'on voit apparaître ponctuellement dès 1292, paraissent permanents. Outre la fonction judiciaire qu'on va décrire dans un moment et qui ne paraît pas la plus importante de leurs prérogatives, ils gèrent et défendent les communaux et représentent la communauté face au prieur. Encore à la fin $\mathrm{du} \mathrm{XV}^{\mathrm{e}}$ siècle, l'assemblée générale de la communitas continue de se réunir régulièrement et de prendre ses décisions à l'unanimité. 
11 C'est dans la sentence arbitrale de 1330 qu'on trouve la plus ancienne allusion à l'exercice de la haute justice par les prud'hommes de Chamonix. Ce texte le considère comme un point de coutume bien établi et que nul ne songe à remettre en cause, le débat portant seulement sur le conseil juridique que le prieuré est tenu de fournir aux prud'hommes quand ils jugent les malfaiteurs : le prieur rechigne à en assurer les frais, qui ne sont pas négligeables, puisque ce conseil doit être recruté extra terram. Les arbitres se contentent d'ailleurs de prescrire que sur ce point l'on s'en remette à la coutume ${ }^{16}$.

12 Il faut attendre ensuite 1414 pour voir la question revenir au jour, d'ailleurs à l'occasion d'un procès d'animal, en l'occurrence celui d'une truie responsable de la mort d'un enfant. Les hommes de Chamonix prétendent que la sentence leur revient. Nous devons à la méfiance du prieur Antoine de Saint-Amour (1402-1417) une très précieuse enquête sur ce point de coutume qu'il n'avait jamais vu s'appliquer depuis douze ans qu'il occupait sa charge. Il fit donc interroger Hugues Bottolier, de Servoz, damoiseau, et surtout noble Ramus de Montfort, de Saint-Gervais, qui était châtelain de Chamonix sous le prieur précédent. Du temps qu'il exerçait, les prud'hommes de Chamonix avaient condamné un voleur au pilori, au fouet et au bannissement ; ils avaient acquitté en revanche un habitant de Saint-Gervais qu'on n'avait pu convaincre de vol; ils avaient, enfin, condamné à mort une truie qui avait arraché l'oreille d'un enfant. Il est à noter qu'Antoine de Saint-Amour ne remet pas vraiment en cause les prérogatives judiciaires de la communauté. Il proteste simplement qu'il veut s'informer préalablement sur les droits de ses dépendants pour éviter que ceux de son monastère ne soient lésés ${ }^{17}$.

13 Jacques de Crescherel en revanche, qui lui succède de 1420 à 1439, tente de s'y attaquer dès le commencement de son priorat, par le biais du conseil juridique qu'il est tenu de fournir aux prud'hommes. Dans une sentence arbitrale rendue en 1421 par l'official de Genève, il parvient à faire insérer que les avis du conseil qu'il aura nommé seront contraignants pour les prud'hommes. Les représentants de la communauté protestent aussitôt d'une erreur et il faut à nouveau choisir des arbitres pour régler le cas ${ }^{18}$. Leur sentence n'est pas conservée, mais la suite des événements montre qu'elle fut favorable à la communauté. En effet, nous voyons par la suite les Chamoniards rendre librement diverses sentences, en matière de sorcellerie notamment ${ }^{19}$.

Ce n'est qu'à la fin du siècle que le prieur Guillaume $\mathrm{I}^{\mathrm{er}}$ de La Ravoire (1439-1487) ${ }^{20}$ se décide à attaquer de front le droit des Chamoniards à juger en matière criminelle. À une date inconnue, mais certainement postérieure à la victoire sans appel qu'en 1485 il avait remporté sur ses hommes en brisant une grève des dîmes ${ }^{21}$, il tente de récupérer par voie de justice l'intégralité des droits de juridiction, en intentant à la communauté un procès devant le vicaire de l'abbaye Saint-Michel de la Cluse ; il est débouté, tant les droits des Chamoniards sont bien fondés par la coutume orale et les témoignages écrits. En 1487, il se démet de la charge priorale en faveur de son fils Jacques (1487-1502), lequel fait appel à Rome et obtient une sentence plus favorable à ses intérêts ; mais la communauté fait appel à son tour, et la cause est encore pendante devant la Rote lorsqu'en 1493 les deux parties se décident à transiger à l'amiable. Jacques de La Ravoire renonce purement et simplement à toutes ses prétentions. Le texte de la transaction ne fait d'ailleurs que réaffirmer et préciser une coutume bien attestée par les documents antérieurs ${ }^{22}$. 
15 Il était temps, selon toute apparence, que les deux parties se missent d'accord, car le mandement de Chamonix devenait un sanctuaire pour les criminels ; il était notable à cette époque que le prieur y tolérait « les malffactors que sunt de [lui] sujet et autres estraniers $»^{23}$. Deux véritables tueurs à gages, peut-être issus des rangs des "Robes rouges » qui avaient écumé le Faucigny à l'été et à l'automne 1492, y vivaient en toute impunité, au grand dam de l'évêque de Sion et de son bailli, qui les recherchaient pour un assassinat commis en Valais ${ }^{24}$. Lorsqu'en juin 1494 Jacques de La Ravoire se décide enfin à les poursuivre, ils ont évidemment disparu. Mais à cette occasion, il s'engage devant ses hommes à maintenir bonne justice dans la vallée et à réprimer les crimes qui s'y commettent; en échange, les syndics promettent au nom de la communauté de lui prêter appui ${ }^{25}$. Cet apaisement n'est pourtant pas du goût de tous les Chamoniards : Michel Bertoud, un de ceux qui avaient pris une part active à la grève des dîmes de 1485, tente d'assassiner le prieur. En 1495 pourtant, ce sont les prud'hommes de Chamonix qui le condamnent à mort ${ }^{26}$. C'est la dernière sentence que nous les voyons rendre avant 1519, date de la suppression du prieuré de Chamonix et du rattachement de son mandement à la collégiale voisine de Sallanches. C'était l'œuvre de Guillaume II de La Ravoire, frère et successeur de Jacques, qui se résolvait à échanger sa charge priorale contre un canonicat plus tranquille à Sallanches, en raison de « la grosse poyne et travail qu'il avoit à governer ses subgestz dudit lieu de Chamonix $»^{27}$.

Abondance

16 Les libertés et franchises des habitants de la vallée d'Abondance ne concernent que le territoire occupé par les deux paroisses du temporel initial de l'abbaye, celles d'Abondance et de la Chapelle des Frasses. La paroisse voisine de Vacheresse notamment, dont le terroir occupe la basse vallée de la Dranse d'Abondance, n'en bénéficie pas, bien que les abbés d'Abondance y aient acquis depuis le XIII ${ }^{\mathrm{e}}$ siècle l'essentiel des droits seigneuriaux, y compris la haute justice ${ }^{28}$.

17 L'histoire de la communauté des habitants de la vallée d'Abondance est moins bien connue que celle de la communauté de Chamonix, surtout dans ses commencements. En 1325 est mentionnée pour la première fois l'universitas hominum de la paroisse de la Chapelle des Frasses. En conflit avec l'abbé d'Aulps sur l'interprétation de plusieurs points de coutume, elle se donne pour l'occasion des procuratores et scindici auxquels elle confie la mission de formuler ses doléances. L'abbé nomme à son tour deux procuratores en la personne de François de Valliège, clerc, et de Guillaume de Châtillon, damoiseau. Il les charge d'examiner point par point les revendications de la communauté et de juger s'ils jugent bon d'y satisfaire. La procédure est donc différente de celle qui est habituellement suivie à Chamonix : il ne s'agit pas d'un arbitrage, mais d'une décision souveraine de l'abbé, représenté par ses procureurs ${ }^{29}$. En fait, les décisions prises concernent les habitants des deux paroisses du mandement d'Abondance. Même si c'est le plus ancien qui soit conservé, ce n'est pas le premier document écrit réglant les rapports entre les habitants de la vallée et les chanoines. Ce texte cite d'ailleurs une convention passée en 1279, dans laquelle l'abbé réglait un différent qui nous reste inconnu, en l'échange de l'engagement pris par ses hommes de ne pas aller se faire recevoir comme bourgeois dans une ville franche ${ }^{30}$. L'abbé évoque également, pour affirmer qu'elles restent toujours en vigueur, les littere olim facte inter nos abbatem et conventum predictos et predecessores nostros ex una parte, et predictos homines dicte vallis ac antecessores eorum ex altera ${ }^{31}$. C'est pourtant la sentence de 1325 que les habitants de la vallée font confirmer par le pape Martin V en $1417^{32}$. La justice y occupe une certaine 
place, et l'on y affirme notamment que le juge (causidicus) du monastère doit fixer le montant des bans, c'est-à-dire des amendes, sur le conseil des prud'hommes du lieu ${ }^{33}$. Mais qui dit bans dit moyenne ou basse justice. Du droit des paysans d'Abondance à connaître les causes criminelles, il n'est pas question dans ce texte.

En 1399, le comte Amédée VIII de Savoie, en réponse à une supplication des hominum, incolarum et habitatorum vallis Habundantie, les avait déjà confirmés, mais sans se référer à aucun texte, in omnibus eorum bonis usibus, quonsuetudinibus et libertatibus ${ }^{34}$. Au début $\mathrm{du} \mathrm{XV}^{\mathrm{e}}$ siècle, les conflits se multiplient entre les habitants et les chanoines, auxquels on tente de remédier par un arbitrage de la duchesse de Savoie Marie de Bourgogne en 1419, par une transaction amiable en 1420, par un nouvel arbitrage en 1425. Seul ce dernier, rendu par Amédée VIII de Savoie, a été conservé35. Il tente de mettre d'accord Guillaume de Lugrin, abbé d'Abondance, et les homines ac communitatem vallis Abudancie. À cette date donc, la communitas a indubitablement dépassé le cadre de la paroisse pour s'étendre à la vallée tout entière, comme à Chamonix. Comme à Chamonix encore, son unité vient de la possession collective des biens communaux, en l'occurrence les alpages de la vallée. L'objet de l'arbitrage est d'ailleurs l'auciège dû pour ces alpages, que les habitants refusent alors de payer, arguant que les chanoines leur refusent l'accès à une de leurs montagnes et qu'ils ne leurs fournissent pas, contrairement à la coutume, les chaudières nécessaires à la fabrication du fromage ${ }^{36}$. L'arbitrage d'Amédée VIII, qui se contente à peu près de recommander que chacun fasse un effort et qu'on s'en remette à la coutume, ne suffit pas à apaiser les tensions.

Bien au contraire, les hommes d'Abondance refusent après 1425 d'acquitter les dîmes et les terrages, et les lite, querele et contemptiones avec les chanoines se multiplient, au sujet des lods et ventes, de la mainmorte, de la construction des moulins, etc. ${ }^{37}$. Le conflit s'envenime au point qu'en 1429, un métral ${ }^{38}$ de l'abbaye, du nom de Berbillaux, est assassiné. Le meurtrier présumé est un certain Mermet Burnier. Les officiers de l'abbé l'arrêtent et le mettent en prison, où on le retrouve pendu. L'abbé affirme qu'il s'est suicidé et réclame que justice soit faite néanmoins contre ses complices, dont un est également emprisonné, un autre en fuite. Les hommes d'Abondance prétendent au contraire que les officiers de l'abbaye sont responsables de sa mort et qu'ils doivent être poursuivis ${ }^{39}$. Surtout, ils se plaignent que Burnier ait été indûment soustrait à leur juridiction. C'est la première fois qu'on les voit affirmer leur droit de juger les criminels ${ }^{40}$. C'est encore à Amédée VIII qu'on demande d'arbitrer le conflit. Entouré de quatre assesseurs, il rend une sentence arbitrale en avril $1430^{41}$. Il y déclare qu'il n'est pas vraisemblable que les officiers de l'abbaye aient assassiné Mermet Burnier, et impose aux hommes d'Abondance un silence perpétuel sur ce point. Mais comme par sa mort justice a été en quelque sorte rendue à Berbillaux, les poursuites contre ses complices doivent être abandonnées, sauf celles engagées contre celui qui, par sa fuite, a reconnu son crime. Cet homme, du nom de Quinclet, est banni pour cinq ans du mandement d'Abondance ${ }^{42}$. Quant au droit de juger les criminels, les arbitres reconnaissent qu'il appartient en première instance aux prud'hommes (probi homines) d'Abondance, également qualifiés de coutumiers (consuetudinarii). Mais les condamnés peuvent en appeler à l'abbé, qui dispose pour cet objet d'un juge des appellations ${ }^{43}$. Cette sentence limite par ailleurs l'usage de la procédure inquisitoriale et celui de la prison préventive, sans lever pourtant toutes les ambiguités, comme nous le verrons. Outre les questions de justice, elle traite de tous les points controversés. Dans l'ensemble très favorable aux habitants de la vallée, puisqu'elle adoucit notamment le régime de la mainmorte, elle est considérée par eux comme la nouvelle charte de leurs 
franchises. Ils ont donc soin, lorsqu'en 1439 le duc Amédée VIII cède le pouvoir à son fils Louis, de la faire confirmer par ce dernier ${ }^{44}$. La communauté d'Abondance en reçoit une nouvelle vigueur. C'est notamment à partir de cette époque que les quatre syndics apparaissent permanents.

La justice ne cesse pas pour autant d'être une pomme de discorde entre les coutumiers et l'abbé. En 1445, le juge de l'abbaye condamne à la pendaison un voleur du nom de Berthet Bullandaz. Ce dernier est originaire de Vacheresse et c'est dans cette paroisse qu'il a commis ses crimes. Les coutumiers d'Abondance n'avaient donc nullement à connaître de sa cause. Mais le jugement a eu lieu dans la paroisse d'Abondance, plus particulièrement au lieu-dit le Pas d'Abondance, là où ils avaient accoutumé de rendre leurs sentences. Ils estiment donc qu'à ce titre la cause devait leur revenir. Il faut que l'abbé affirme, par une transaction amiable passée en octobre 1445, que l'affaire ne créera pas de précédent nuisible aux coutumes de la vallée et que dorénavant toutes les sentences rendues au Pas d'Abondance seront du ressort des coutumiers ${ }^{45}$. Si cet incident, somme toute mineur, prend alors une telle importance, c'est qu'il se greffe sur un confit plus grave, relatif à l'usage persistant de la procédure inquisitoriale et de la prison préventive par la justice de l'abbaye. Sur ce point aussi, l'abbé se trouve contraint de céder, comme nous le verrons. En tout cas, cette dernière transaction rétablit durablement la paix. Par la suite, nous voyons les coutumiers rendre diverses sentences jusqu'à la mi-XVI siècle $^{46}$.

21 Les points de contact entre l'histoire des deux communautés d'Abondance et de Chamonix sont donc nombreux: apparition tardive (fin XIII ${ }^{\mathrm{e}}$ siècle) de traces écrites des «franchises ", la rédaction de la coutume ne concernant que les point controversés, à l'occasion du règlement amiable des conflits par transaction ou par arbitrage ; apparition plus tardive encore (mi-XVe siècle) d'institutions permanentes, les communautés semblant se contenter pendant longtemps de nommer des procureurs, éventuellement qualifiés de syndics, pour régler des affaires particulières; prise de conscience progressive d'intérêts communs à toute une vallée et dépassement du cadre de la paroisse, par le biais de la gestion collective des biens communaux, principalement des alpages.

Quant à l'exercice de la justice criminelle par les tribunaux paysans, c'est un point de coutume qui n'apparaît dans la documentation que lorsqu'il fait l'objet d'un conflit, mais dans les deux vallées il est manifestement plus ancien que les premières mentions écrites qu'on en possède. À Abondance, il n'est jamais vraiment contesté par les abbés. Ce n'est qu'à l'occasion de la mort par bavure ou accident de Mermet Burnier et de la condamnation de Berthet Bullandaz que les coutumiers ont pu croire leurs privilèges menacés. À Chamonix en revanche, il a fait l'objet d'une véritable offensive de la part de plusieurs prieurs, Jacques de Créscherel dans les années 1420, et surtout les prieurs de La Ravoire à la fin du XV siècle. Mais il était si bien consacré par la coutume que ces attaques ont été vaines.

Il s'agit maintenant de voir comment on en usait dans l'une et l'autre vallées.

L'exercice de la justice criminelle par les tribunaux paysans : modalités et limitesModalités

24 À qui revient l'exercice de la justice criminelle? Aux homines, aux probi homines à la communitas, dit-on à Chamonix; aux homines, aux probi homines, aux consuetudinarii, affirme-t-on à Abondance. Dans ce contexte, ces termes désignent dans chacune des 
deux vallées la communauté prise dans son ensemble. Les sentences conservées pour le $\mathrm{XV}^{\mathrm{e}}$ siècle montrent comment ce principe était mis en œuvre dans la pratique.

Voici par exemple en 1470 le jugement du Chamoniard Jean Pecluz, convaincu d'adultère, d'inceste, d'agression contre le curé de Vallorcine, d'évasion de la prison de Chamonix et de voies de fait contre les officiers du prieur ${ }^{47}$. Par les soins du vicechâtelain de Chamonix, il est extrait de la prison du prieuré où on était parvenu à le remettre, et amené à la «banche » de la cour (bancha curie), située près du monastère, coram [...] probis hominibus, videlicet majori parte proborum hominum dicte communitatis Campimuniti. Les prud'hommes sont probablement debout mais, assis à la banche proprement dite, siège Petrus de Lavancherio, consindicus, judex et cognitor [...] per dictam communitatem et probos homines ejusdem communitatis electus, deputatus, constitutus et ordinatus. Le procureur fiscal du prieuré se présente à lui, lui demandant de bien vouloir de dicto Johanne Pecluz justiciam ministrare. Pierre du Lavancher se fait lire par un notaire l'acte d'accusation (processus), rédigé en langue vulgaire (lingua layca). Il interroge ensuite l'accusé, lui demandant s'il reconnaît les faits qui y sont relatés. Après la réponse affirmative de ce dernier, prononcée coram populo ibidem existente, il délibère avec Martin Sostion, jurisperitus, conseil juridique fourni par le prieur. La délibération, dont le notaire présent a rédigé un instrument public, porte d'abord sur les faits : ceux qui sont avoués sont considérés comme avérés. Relativement à l'ultime accusation, celle de complot contre le prieur de Chamonix, il n'y a ni aveu ni preuve. Vient ensuite le conseil juridique proprement dit, duquel il ressort que chacun des faits reconnus par l'accusé est à lui seul passible de la peine de mort. Puis Pierre du Lavancher rend son verdict, qui suit l'avis du conseil, puisque Jean Pecluz est condamné a avoir le poing tranché avant d'être pendu. Il rend le condamné au vice-châtelain et lui demande d'exécuter la sentence.

Transportons-nous à Abondance, où nous voyons en 1476 le procureur fiscal de l'abbaye, qui se trouve être aussi son administrateur en l'absence de l'abbé commendataire, comparaître sous la halle du Pas d'Abondance pour demander que justice soit faite contre Jean Marollaz, malfaiteur en cavale, réfugié et repris dans la vallée. Marollaz comparaît à son tour, demandant à être relaxé. Siègent en ce jour 109 prud'hommes, nommément cités dans l'instrument public de la sentence. Les quatre syndics viennent en tête de liste, mais rien ne dit qu'ils occupent une position éminente. Pour le reste, la procédure ressemble fort à celle qu'on suit à Chamonix : lecture en langue vulgaire de l'acte d'accusation et des réponses de l'accusé; délibération des prud'hommes cum peritis in jure et consuetudine; enfin énoncé du verdict par le premier nommé des syndics : convaincu seulement de s'être évadé des prisons de l'abbaye d'Agaune, les autres chefs d'accusation n'étant pas retenus faute de preuves, Marollaz est banni pour un an du mandement d'Abondance. Le temps, pour lui, d'aller se faire pendre ailleurs ${ }^{48}$.

27 Apparemment, la plus notable différence de procédure avec le cas chamoniard est qu'ici il n'y a pas un seul juge, mais 109 ! Certes, le résultat de la délibération est rapporté par un des syndics, mais la sentence émane bien de l'ensemble des coutumiers présents. D'ailleurs elle engage aussi les absents, réputés consentants: c'est la communauté d'Abondance qui tranche à l'unanimitét ${ }^{49}$. En fait, la différence est de forme plutôt que de fond. Car en droit, à Chamonix aussi ce sont bien tous les membres de la communauté qui, pris collectivement, sont juges (judices et cognitores) en matière criminelle ${ }^{50}$. Concrètement, comme ils ont l'habitude de le faire en toute sorte de 
matières, ils constituent un procureur chargé de les représenter pour cette tâche particulière. Mais c'est bien de eorum consensu et voluntate qu'il rend sa sentence, et d'ailleurs in ipsorum presencia. Comme dans les assemblées de la communauté, tous n'ont pas fait le déplacement mais les absents, là encore, sont réputés consentir car dans les sentences criminelles comme dans toutes les décisions prises par la communauté, les prud'hommes doivent être unanimes et concordes ${ }^{51}$. Si la langue vulgaire est employée pour la lecture de l'acte de l'accusation et le prononcé de la sentence, c'est pour que l'un et autre soient compris de l'accusé, mais aussi des juges. Outre les problèmes pratiques que peut poser une délibération à plus de cent prud'hommes, la démocratie directe pratiquée à Abondance fait courir le risque à la communauté de mettre sur la place publique ses éventuelles divergences interne. À Chamonix en revanche, le consensus s'exprime dans le choix préalable du juge délégué. Celui-ci reçoit d'ailleurs des lettres de sauvegarde, de sorte que ceux qui seraient insatisfaits de la sentence rendue sachent que ce n'est pas la seule responsabilité du juge qui est engagée, mais celle de toute la communautées2.

Il est notable qu'en aucun cas les prérogatives judiciaires ne sont liées à la charge syndicale. À Abondance, nous venons de voir, le syndic qui énonce la sentence n'est que le porte-parole des probi homines. À Chamonix, il se trouve que dans l'exemple que nous avons choisi, Pierre du Lavancher est syndic par ailleurs. Mais c'est un cas unique dans les sentences conservées et ce n'est pas en tant que tel qu'il rend la justice. Si d'aventure les syndics siègent aux côtés du juge à la bancha curie, c'est seulement pour y représenter la communauté et manifester d'avantage son accord avec celui qu'elle a délégué, lequel rend seul le jugement ${ }^{53}$.

Mobilisant à tout le moins plusieurs dizaines de judices et cognitores, ces sortes de procès devaient présenter un caractère spectaculaire. Ceci posé, il faut convenir pourtant que les prérogatives judiciaires des prud'hommes était très étroitement bornées.

Limites

Certes, dans les deux mandements nous voyons les prud'hommes maîtres de l'issue des procès criminels, et ce n'est pas rien. Mais il faut tout de même remarquer qu'ils n'interviennent que ponctuellement, dans une procédure pour le reste entièrement contrôlée par les officiers seigneuriaux. Les exposés les plus systématiques de la coutume, qui sont à Abondance la sentence de 1430 et à Chamonix la transaction de 1493, l'affirment sans ambiguitéé ${ }^{54}$. Les pièces de procédure conservées le montrent non moins clairement : l'instruction est menée par le châtelain où son lieutenant, sous le contrôle et parfois avec l'intervention directe du juge ordinaire. Rédigé par le notaire de la cour (clericus curie), l'acte d'accusation est soutenu devant les prud'hommes par le procureur fiscal. À moins de relaxe, l'exécution de la sentence revient au châtelain.

31 À Abondance, du point de vue des prud'hommes nous sommes en pleine procédure accusatoire : sauf effet de sources, ils ne jouent aucun rôle dans l'instruction. Le procureur fiscal et l'accusé sont comme à égalité devant eux ${ }^{55}$. À Chamonix, selon les termes de la transaction de 1493, les syndics ont le droit de désigner trois prud'hommes pour assister aux interrogatoires et y participer s'ils le souhaitent, après toutefois que l'accusé ait été laissé dix jours entre les seules mains des officiers du prieur ${ }^{56}$. À la fin des procès verbaux des interrogatoires de Jean Pecluz, deux à cinq prud'hommes sont cités comme témoins, mais ils ne semblent pas avoir pris la parole. Il est notable que Pierre du Lavancher, qui le condamnera finalement au nom de la communauté, ne se trouve pas parmi eux ${ }^{57}$. 
(1) procédure, l'accusé a la possibilité de mettre fin aux poursuites engagées contre lui en acceptant de composer avec le prieur ou l'abbé. La composition en matière pénale est une pratique extrêmement répandue en Savoie et en Dauphiné aux XIV et $\mathrm{XV}^{\mathrm{e}}$ siècles. Elle est commune aux justices seigneuriales et princières; ces dernières ont d'ailleurs laissé des documents comptables enregistrant la perception d'innombrables banna concordata ${ }^{58}$. Nos deux vallées ne font pas exception à la règle. Dans toutes les procédures engagées, les officiers seigneuriaux négocient avec l'accusé en même temps qu'ils mènent l'instruction. À Chamonix, les dix jours d'incarcération spécifiés par la transaction de 1493 sont explicitement réservés à cet effet, l'absence de témoins extérieurs étant propre à faciliter la négociation. Mais cette absence même pouvait être dangereuse, et la transaction précise que le prieur doit résister à deux tentations opposées : négliger les intérêts de la victime, dans le cas d'un accusé trop pressé de composer; employer la torture pour amener à composition un accusé réticent ${ }^{59}$. À Abondance d'ailleurs, les prud'hommes se plaignaient en 1325 que le métral leur extorquât « des bans obscurs mal négociés ${ }^{60}$. La composition, issue la plus commune des procédures pénales en matière de basse justice ${ }^{61}$, n'était apparemment pas rare dans les causes de haute justice. Le chartrier du prieuré de Chamonix a conservé la trace de plusieurs compositions acquittées par des voleurs ou des meurtriers; ce sont habituellement des reconnaissances de dettes, car ces compositions, fort élevées, étaient payées en plusieurs termes ${ }^{62}$. En tout cas, à Chamonix comme à Abondance, les prud'hommes n'avaient l'occasion de rendre la justice qu'en cas d'échec des négociations entre les accusé et les officiers seigneuriaux.

Reste enfin une spécificité chamoniarde, qui est la présence dans le chartrier du prieuré de six sentences criminelles prononcées par le juge ordinaire ${ }^{63}$; présence agaçante car difficile à expliquer : toutes les conditions de droit sont réunies pour que la connaissance de ces causes revienne aux prud'hommes, et pourtant nous ne les voyons pas protester lorsque le juge rend sa sentence. Deux explications sont possibles, qui ne s'excluent nullement. D'une part, on se rappelle que les sentences nécessitaient une unanimité de la communauté ; si celle-ci était impossible à obtenir, par exemple parce que les parents et amis du prévenu prenaient fait et cause pour lui, on peut imaginer que les prud'hommes se trouvaient dans l'obligation de renoncer pour une fois à l'exercice de leur pouvoir judiciaire. Par ailleurs, il est notable que les sentences criminelles prononcées par le juge sont chronologiquement groupées, puisqu'elles se situent toutes entre 1364 et 1431; il n'est pas impossible que les deux ou trois générations encadrant le tournant des $\mathrm{XIV}^{\mathrm{e}}-\mathrm{XV}^{\mathrm{e}}$ siècles aient été moins attentives à ce point de coutume que les précédentes et les suivantes. Il aurait d'ailleurs pu se perdre, puisque c'est précisément le moment où nous voyons les prieurs s'en étonner d'abord, puis le remettre en cause.

Toujours est-il que dans nos deux vallées les prud'hommes ne se trouvaient pas très fréquemment dans le cas de rendre un verdict. En sorte que lorsqu'à la fin du XVe siècle les prieurs de La Ravoire tentèrent de mettre fin à aux privilèges judiciaires des Chamoniards, ils purent affirmer avec quelque vraisemblance que l'exercice de la haute justice par leur juge était la règle et que ce n'était que par exception qu'ils la 
déléguaient à la communauté ${ }^{64}$. Les documents conservés dans le chartrier du prieuré leur eussent donné raison, si les Chamoniards n'avaient fait valoir les différentes sentences arbitrales dont ils avaient bénéficié, notamment celle de 1330.

$\mathrm{Au}$ total, il convient donc de ne pas exagérer le caractère exceptionnel des privilèges judiciaires des communautés de Chamonix et d'Abondance. Pour mieux en mesurer la portée, il convient maintenant de les replacer dans le contexte environnant.

Les libertés des communautés d'Abondance et de Chamonix à la mesure du contexte alpin

Les Alpes nord-occidentales connaissent trois types de seigneurs haut-justiciers. Les princes sont de très loin les plus importants : ce sont les dauphins (jusqu'à 1343), les sires de Faucigny (jusqu'à 1355), les comtes de Genève (jusqu'à 1402), et les comtes puis ducs (1416) de Savoie, ces derniers étendant progressivement leurs domaines de la Chartreuse au Pays de Vaud et du Lyonnais au Piémont, avant de connaitre un relatif repli à la fin $\mathrm{du} \mathrm{XV}^{\mathrm{e}}$ siècle. Viennent ensuite des seigneurs ecclésiastiques, comme le prieur de Chamonix et l'abbé d'Abondance, ou encore, pour se tenir dans les environs immédiats de ces deux monastères, les abbés d'Aulps, de Saint-Maurice d'Agaune et de Sixt, les évêques de Sion et de Genève. Il faut enfin compter avec quelques seigneurs laïcs de moindre importance.

38 Les garanties judiciaires prévues par les chartes de franchises rurales et urbaines accordées par ces différents seigneurs peuvent être commodément ramenées à trois points: 1) la participation des justiciables au pouvoir normatif;2) les garanties procédurales ; 3) l'association des justiciables à l'exercice de la justice.

La participation des justiciables au pouvoir normatif

Dans plusieurs communautés urbaines et rurales des Alpes nord-occidentales, les justiciables ont un certain accès au pouvoir réglementaire, en ce sens qu'ils sont autorisés par leurs seigneurs à intervenir dans la fixation des bans, terme à comprendre cette fois au sens de pénalités prévues par le droit pour une infraction donnée ${ }^{65}$.

Dans les pays romands, une participation des bourgeois au pouvoir normatif aux côtés du seigneur est attestée dès la fin du XIII ${ }^{e}$ siècle dans certaines cités pourvues de franchises, comme Moudon (Pays de Vaud) ou Sion (Valais). Elle se généralise au début $\mathrm{du} \mathrm{XV}^{\mathrm{e}}$ siècle, la coutume vaudoise l'étendant même aux communautés rurales. Dans le même temps, les législations urbaines se passent de plus en plus d'une confirmation princière ${ }^{66}$.

41 Le Dauphiné et la Savoie fournissent aussi, mais en petit nombre, des chartes de franchises urbaines attestant une participation des bourgeois à la tarification des bans. Dans la première moitié du XIII ${ }^{\mathrm{e}}$ siècle, les consuls d'Embrun (Dauphiné) font faire des criées relatives à la police urbaine et fixent des bans à l'encontre des contrevenants ${ }^{67}$. En 1285, la charte de Seyssel (Avant-pays savoyard) affirme le pouvoir législatif des bourgeois en une formule aussi ferme que lapidaire: Quodcumque bannum burgenses volunt ponere, castellanus debet conceder ${ }^{68}$. De même, la première charte de Flumet (Faucigny) affirme en 1228 : Quodcumque bannum imponetur cum conjuratorum consilio ${ }^{69}$.

Bien plus nombreuses sont les chartes de franchises urbaines qui se contentent de consacrer un certain nombre d'articles à fixer le tarif des bans. La présence de tarifs d'amendes dans une charte de franchise suppose que l'autorité seigneuriale et les bourgeois se soient mis d'accord pour les y insérer, soit qu'ils aient fait l'objet d'un 
consensus, soit au contraire que leur mise par écrit vise à mettre fin aux les conflits les concernant. On considère généralement que ces sortes de tarifs visent essentiellement à fixer des maxima, et de la sorte à mettre un frein à l'arbitraire seigneurial, dans le cadre de justices que leurs détenteurs regarderaient essentiellement comme des sources de profits ${ }^{70}$. Mais on est frappé par le grand nombre d'infractions (plus de la moitié dans la plupart des chartes de franchises savoyardes) qui sont sanctionnées par le bannum grossum, dont le poids minimum est de $60 \mathrm{~s}$. Outre son importance ${ }^{71}$, ce montant est remarquable par son caractère manifestement symbolique. Issu de la tradition carolingienne, il est indépendant des variations de la valeur réelle du sou dans les différentes monnaies utilisées dans les Alpes. D'ailleurs, le chiffre symbolique de 60 s'applique aussi bien à des livres qu'à des sous. Dans les franchises d'Aoste par exemple (c. 1191), l'effraction d'une maison particulière, l'agression d'un homme à l'intérieur de son domicile, l'usage d'un arc ou d'une arbalète dans les limites de la franchise ou encore la destruction des ponts sont passibles d'une amende de 60 livres $^{72}$. De telles sommes vont évidemment bien au-delà des facultés réelles de la plupart des délinquants potentiels, et l'on ne voit pas que ces maxima aient le moins du monde constitué une protection pour les justiciables. On dira, avec raison, que certains banna grossa ont pu venir en rachat d'un châtiment corporel. Mais puisqu'il était bien impossible aux justiciables de les acquitter, la protection restait totalement illusoire.

À notre sens, il faut donc dire au contraire que les infractions sanctionnées par un bannum grossum sont celles qu'on souhaite réprimer sévèrement, presque à l'égal de celles qui sont passibles d'une peine afflictive. Les données rassemblées par Ruth Mariotte-Löber à partir des franchises concédées par les comtes de Savoie ${ }^{73}$ montrent que les banna grossa répriment essentiellement deux catégories d'infractions. Premièrement les attaques caractérisées contre les intérêts du comte ou les attributs de sa souveraineté : fraude fiscale, refus de partir en chevauchée, etc.; en les réprimant sévèrement, le prince savoyard affirme sa souveraineté sur la ville. En second les infractions propres à briser la paix et la confiance entre les habitants de la cité : l'adultère et le rapt, la violence sous ses formes les plus graves à l'exclusion du meurtre (effusion de sang, agression à l'intérieur du domicile et incendie volontaire) et l'utilisation de fausses mesures; cette deuxième catégorie d'infractions a dû faire l'objet d'un consensus avec les bourgeois, intéressés comme le prince au maintien de la paix dans la cité. Ces bans, comme d'ailleurs les bans de moindre importance (banna minuta) sont très stéréotypés et ils attestent des phénomènes de filiation entre chartes. Il n'en est guère qui portent sans conteste la marque de l'intervention des bourgeois dans leur fixation, et l'on peut seulement évoquer un consensus global.

Celui-ci devait être d'autant plus facile à obtenir que depuis la fin du XIII ${ }^{e}$ siècle au moins, la pratique de l'arbitraire pénal (au sens positif du terme) et celle de la composition sont cause que les bans effectivement levés sont incomparablement moins lourds que ceux qui sont spécifiés dans les franchises. Il n'empêche qu'en Dauphiné ces derniers, bien loin de baisser, c'est-à-dire de se rapprocher de la pratique, ont tendance à s'élever avec le temps ${ }^{74}$.

Certaines chartes rurales présentent bien plus de signes d'une participation concrète des paysans à l'élaboration des bans. Celle-ci est d'ailleurs attestée dès avant la concession des premières franchises écrites. En Val d'Aoste par exemple, certaines communautés rurales ont élaboré en accord avec leur seigneur ce qu'Ezio-Émeric Gerbore nomme des « règlements de police", qui concernent essentiellement les bans 
champêtres. Si les communautés étaient associées à la rédaction de ces statuts, leur application revenait au tribunal seigneurial. On a conservé ceux des communautés de Châtillon et Fénis. Rédigés en 1288, ils sont antérieurs aux plus anciennes chartes de franchises rurales conservées en Val d'Aoste ${ }^{75}$.

À l'échelle européenne, les franchises rurales apparaissent fort tardivement dans les Alpes nord-occidentales, puisque on n'en trouve que depuis la fin du XIII ${ }^{e}$ siècle. C'est que dans ces régions les chartes de franchises prennent la suite des plaids généraux, que par leur existence même elles font tomber en désuétude. Institution propre aux communautés dont les rapports avec l'autorité seigneuriale sont régies par une coutume orale, le placitum generale s'est tenu dans certaines seigneuries alpines jusqu'au commencement du XIV siècle. Souvent annuel, il réunit le seigneur et ses hommes pour un record de coutumes et des assises générales. À partir de l'exemple de la Suisse romande, on a très bien montré ${ }^{76}$ que le plaid général a trois fonctions si étroitement imbriquées qu'elles sont inséparables: 1) rappeler aux justiciables les règles du droit local, que nul n'est censé ignorer, ce faisant manifester la puissance du seigneur justicier ; 2) rappeler aussi les limites coutumières de cette puissance, et éventuellement en instaurer de nouvelles ; 3 ) organiser le nécessaire dialogue entre le seigneur et ses hommes, dans le domaine économique notamment.

Plus tard, certaines chartes de franchises rurales portent la marque de ce dialogue, en ceci qu'elles contiennent des articles relatifs aux bans ruraux qui sont si circonstanciés qu'il est manifeste que l'initiative en revient aux paysans. Ainsi par exemple de la charte accordée en 1324 par l'abbé de Saint-Maurice d'Agaune à la communauté de Salvan (Valais occidental) ${ }^{77}$. Les bans qui sanctionnent la fraude fiscale sont à n'en pas douter d'origine seigneuriale. Mais d'autres interdisent la montée en alpage avant les dates coutumières, sanctionnent la divagation du bétail, répriment l'occupation indue des châbles par où l'on a coutume de débarder le bois, organisent la mise en défens estivale des prés de fauche de fond de vallée, interdisent la déforestation au-dessus des villages, dans le but manifeste de les préserver des avalanches. Ceux-là visent à défendre les intérêts communautaires contre la tentation sans cesse renaissante de l'individualisme agraire ; ils collent de très près à la situation locale et n'ont pu être institués qu'à la demande des autorités de la communauté.

A contrario, il arrive aussi qu'en concédant des franchises écrites, tel seigneur se réserve le droit de révoquer certains points d'une réglementation communautaire antérieure. C'est attester que celle-ci existait dès avant la concession des franchises et qu'elle continuera du reste d'exister, mais sous le contrôle seigneurial ${ }^{78}$.

Les différents textes qui contiennent les franchises de Chamonix et d'Abondance ne consacrent aucun article à la tarification des bans. Bien plus, on a conservé pour le mandement de Chamonix les textes de trois "criées » effectuées aux portes des églises paroissiales de Vallorcine et Chamonix en 1417, 1435 et $1440^{79}$. Comme les tarifs de bans des chartes de franchises urbaines savoyardes, elles répriment essentiellement la fraude fiscale, la violence et les infractions contre la moralité publique. Aucun article n'en peut être isolé qui porte la marque d'une initiative paysanne. Et d'ailleurs il s'agit clairement de documents qui émanent de l'autorité seigneuriale. C'est le prieur qui établit souverainement la liste des infractions et le montant des amendes.

50 En 1483 , les syndics de la communauté font interdire par criée aux Chamoniards d'amodier des dîmes du prieuré. Dans le procès qui s'ensuit, ils doivent avouer qu'ils ont usurpé l'officium magistratus, terme qui dans ce conteste désigne clairement le 
pouvoir réglementaire. Et l'un d'eux doit d'ailleurs reconnaître quod nunquam audivit fieri cridas, nisi ex parte domini Campimuniti ${ }^{80}$. Ce n'est pas que la communauté ne prenne jamais de décisions : dans les mêmes années on voit son assemblée générale se réunir in faciendo plura arresta pro ducendo bestias in montibus ${ }^{81}$. Mais jamais le prieur n'intègre ses décisions à sa législation pénale. Pour les faire respecter, elle devra attaquer les contrevenants au civil, devant le juge ordinaire du prieuré.

51 Les habitants de Chamonix, non plus, semble-t-il, que ceux d'Abondance ne participent donc le moins du monde à la fixation des bans dans les deux juridictions. En ce sens, leurs franchises sont en retrait par rapport à celles de bien des localités rurales et urbaines. Il n'en va pas de même en ce qui concerne les garanties relatives à la procédure.

Les garanties en matière de procédure

Jean-François Poudret a montré que dans les chartes de franchises romandes, les trois garanties procédurales les plus communes sont: 1) le droit pour tout accusé ou défendeur d'être jugé par le juge de son domicile ; 2) l'interdiction faite à la justice seigneuriale de poursuive d'office ; 3) l'impossibilité d'incarcérer une personne apte à fournir caution, sauf si elle est accusée de crime de sang ${ }^{82}$. Ces remarques sont valables pour l'ensemble des Alpes du nord, comme le montrent les études de Ruth MariotteLöber sur la Savoie et de Pierre Vaillant sur le Dauphiné83.

Ces trois garanties sont évoquées par les arbitrages chamoniards. Celui de 1292 limite la poursuite d'office à propos des adultères : le juge du prieuré ne pourra plus enquêter à leur propos sans une plainte initiale, à moins qu'il ne se fonde sur un indice, ce qui lui laisse tout de même une grande marge d'initiative ${ }^{84}$. C'est souvent le propre des sentences arbitrales de vouloir si bien concilier les deux parties sur le moment qu'elles laissent subsister des ambiguïtés lourdes de conflits futurs. L'arbitrage de 1368 est un modèle du genre. Il interdit au prieur de citer ses hommes extra juridictionem Campimuniti, sauf toutefois in casum necessitatis vel in culpa ipsorum hominum. Il n'est pas plus clair sur l'usage de la prison préventive ; le prieur prétendait pouvoir emprisonner tout accusé dont la culpabilité lui paraissait établie donec concordavisset seu solvisset judicatum. Les syndics affirmaient que cela lui était interdit, du moment que l'accusé pouvait fournir une caution. L'arbitrage leur donne raison, nisi casus talis esset quod cautio de jure non haberet locum ${ }^{85}$. Comme on l'a vu, la question ne sera finalement réglée que par la transaction de 1493, qui réservera l'emprisonnement préventif aux accusés passibles d'une peine corporelle et en fixera la durée maximale à dix jours.

Les habitants de la Chapelle d'Abondance formulent en 1325 des doléances véhémentes : à les en croire, les métraux et les autres familiers de l'abbé ont coutume de les emprisonner arbitrairement; c'est apparemment, comme l'affirme un autre article qu'on a déjà cité, pour leur extorquer des bans de composition. Les commissaires nommés par l'abbé réservent la prison préventive aux voleurs, meurtriers, traitres ou autres coupables d'un crime passible de la peine corporelle. Accusés d'infractions moins graves, les coutumiers échapperont à l'incarcération, s'ils peuvent fournir une caution $^{86}$.

Un siècle plus tard, il semblerait pourtant que la situation se soit encore dégradée. En 1430 en effet, les prud'hommes d'Abondance peignent à l'intention du duc Amédée VIII un tableau apocalyptique de la situation de leurs libertés: les officiers de l'abbaye, disent-ils, se saisissent quotidiennement de certains d'entre eux et usent de toutes sortes de mauvais traitements afin de les contraindre par la terreur à composer à de 
fortes sommes ${ }^{87}$. Comme de juste, le duc ne manque pas d'ordonner qu'il soit mis fin à ces pratiques. Cependant, il autorise l'emprisonnement préventif dans les cas prévus par la coutume d'Abondance, c'est-à-dire ceux qui sont passibles d'une peine corporelle. Que les officiers du monastère aient alors commis des abus de pouvoir, c'est ce dont on ne peut douter, car le duc nomme deux commissaires chargés de recueillir les plaintes portées contre eux. Mais il faut replacer ces excès dans leur contexte, et se souvenir qu'ils viennent au terme d'une période de cinq ans durant laquelle les redevances seigneuriales n'ont pas été acquittées, non plus que la dîme. Les compositions auxquelles les officiers tâchaient de contraindre les coutumiers par la force regardaient donc certainement des arriérés de paiement.

C'est également à ce contexte qu'il faut rattacher la plainte selon laquelle la justice de l'abbé se saisit d'office, sans dénonciation préalable, contre le droit et la coutume d'Abondance ${ }^{88}$. Amédée VIII consacre cette coutume et interdit les poursuites d'offices, nisi in casibus dumtaxat quibus jure communi permicitur: c'est utiliser le droit écrit (jus communis) à titre supplétif afin de laisser à l'abbé et à ses officiers une nécessaire porte de sortie; car le duc n'envisage pas d'empêcher le juge de l'abbaye de poursuivre d'office les contribuables réticents.

Les prud'hommes, pourtant, ne l'entendent pas de cette oreille, et en 1445 ils réitèrent leur plainte. L'abbé rétorque que ses officiers doivent suffire comme dénonciateurs. C'est être fidèle à la lettre de la coutume mais non à son esprit, et il lui faut transiger sur ce point : un officier de l'abbaye ne sera reconnu comme dénonciateur valable que dans les affaires où il est personnellement intéressé. Toutefois il obtient que soit reprise à la lettre la formule d'Amédée VIII réservant les cas où le droit écrit permet les poursuites d'offices ${ }^{89}$.

On voit que la coutume assure aux habitants d'Abondance des garanties procédurales extrêmement solides; si solides qu'ils ont pu s'en prévaloir pour s'opposer momentanément à la perception des taxes seigneuriales; si solides aussi qu'elles ont pu aller jusqu'à entraver la bonne marche de la justice. C'est au point qu'au commencement $\mathrm{du} \mathrm{XVI} \mathrm{X}^{\mathrm{e}}$ siècle, la vallée, comme celle de Chamonix quelques années plus tôt, devenait un refuge pour toutes sortes de gens sans aveu: en 1525, le mandement se trouve écumé par une bande de "vagabonds et hommes inutiles" contre lesquels nul n'ose porter plainte. Les syndics doivent alors donner au métral la liberté d'enquêter d'office, mais pour trois mois et demi seulement. Ils prennent soin de rappeler longuement la coutume et se réservent le droit d'assister en personne aux interrogatoires. Voilà comment, dans un paisible mandement montagnard, il fallut en venir à déroger des libertés naguères si passionnément défendues, pour éviter qu'elles ne constituent une menace pour l'ordre public et la sécurité des personnes... Le cas n'est d'ailleurs pas unique: en 1348, la communauté vaudoise de Payerne avait dû autoriser le comte de Savoie à enquêter d'office pendant une période de dix ans, pour parer à une recrudescence de la criminalitée ${ }^{90}$.

Pour ce qui est des garanties procédurales, les prud'hommes d'Abondance et de Chamonix bénéficient donc peu ou prou des mêmes libertés que bien des communautés alpines. Il convient maintenant de mesurer l'originalité de leur droit à être jugés par leurs pairs en matière criminelle en le comparant à ce qui se pratiquait dans les régions voisines.

La participation à l'exercice de la justice 
60 En ce domaine, il faut distinguer entre pays de droit écrit et pays coutumiers. Les premiers sont le Dauphiné, le Faucigny, la principauté genevoise sauf la ville même de Genève et la partie non romande de la principauté savoyarde, à laquelle il faut ajouter le bailliage de Chablais dans sa quasi-intégralitép ${ }^{1}$. Les autres pays romands sont régis par la coutume, certaines régions acceptant le droit écrit à titre de droit supplétif ${ }^{92}$.

Dans les pays de droit écrit, une certaine participation des justiciables à l'exercice de la justice pénale est attestée, mais elle reste toujours limitée.

62 En Savoie, Faucigny et Genevois, on ne la rencontre que dans les chartes de franchises urbaines. À Suze (Piémont), cité épiscopale dont les premières libertés connues ont été accordées par le comte de Savoie Amédée III (1103-1148), un tribunal des vicini est attesté en 1170 , dont on ignore les prérogatives ${ }^{93}$. La charte d'Aoste (1191) est plus précise : elle prévoit que les « habitants » fixent les amendes d'un commun accord avec les représentants du comte dans les seuls cas de voies de fait ayant entraîné des blessures $^{94}$, le vol et l'homicide restant à la merci du comte et les autres crimes et délits punissables d'un ban tarifé. En 1228, la première charte de Flumet institue des consuls ad tenendam justiciam recte. Le sire de Faucigny s'engage à venir y tenir son tribunal et à y juger secundum decreta burgensium ${ }^{95}$. Mais une nouvelle charte concédée en 1307 semble bien ramener le bourg de Flumet à la condition des autres villes franches du Faucigny : le dauphin y détient seul tous les droits de justice, moyennant les garanties procédurales classiques ${ }^{96}$. Plus nombreuses sont les chartes urbaines savoyardes genevoises ou faucignerandes qui associent les bourgeois à la seule fixation de la satisfactio accordée à la victime (Chambéry (1232), Saint-Genix (c. 1270), Cluses et Sallanches (1310), etc. $)^{97}$.

63 La situation est comparable en Dauphiné. Aux termes de la charte de 1210, la communauté d'Embrun paraît collaborer avec la justice delphinale «d'une façon d'ailleurs indirecte et qu'il est difficile de préciser ». Le tribunal du dauphin y est assisté «d'une sorte de jury, n'ayant que voix consultative et composé d'officiers municipaux et [...] de prud'hommes ». De même à Gap il existe temporairement au XIII ${ }^{e}$ siècle une juridiction consulaire dont nous ignorons à peu près tout. Quant aux communautés rurales, elles n'exercent que des pouvoirs de simple police; elles perçoivent éventuellement de menues amendes concernant les bans ruraux, parfois à leur profit, comme en Briançonnais, plus souvent au profit du Dauphin (Trièves, Oisans, Gapençais). On voit parfois les consuls, comme en Embrunais, exercer une juridiction arbitrale dans les causes civiles les moins importantes, comme les conflits de mitoyennetés ${ }^{8}$.

Dans les pays de droit écrit, jamais la connaissance des causes pénales ne revient aux seuls bourgeois, que ce soit en matière de haute ou de basse justice. Il n'en va pas de même dans les pays régis par la coutume.

Dans les cours des pays romands en effet ${ }^{99}$, on constate que siègent au côté du juge seigneurial des experts en coutume choisis par lui et qualifiés de prud'hommes (probi homines) ou de coutumiers (consuetudinarii). Chargés de dire le droit, ils ne se contentent pas de donner leur avis, mais ils connaissent véritablement les causes pénales et civiles. Selon les lieux, ils délibèrent en présence du juge seigneurial ou en son absence, mais dans tous les cas celui-ci est lié par leur verdict, n'étant que le rapporteur de leur décision. En pays de Vaud où à Lausanne, ils doivent être unanimes, faute de quoi le juge est libre d'en choisir d'autres. Ailleurs, ils statuent à la majorité. Attesté à la fin du XIII ${ }^{e}$ siècle dans certaines villes pourvues de franchises, comme Moudon (Pays de Vaud) 
ces pratiques paraissent se répandre dans le plat pays dès le XIV ${ }^{\mathrm{e}}$ siècle, pour devenir la règle dans l'ensemble des pays coutumiers.

Les points de ressemblance sont nombreux avec la situation constatée à Chamonix et Abondance, à commencer par l'emploi des termes de prud'hommes et de coutumiers. Certes, dans les pays romands ceux-ci ne sont que les assesseurs du juge, tandis qu'à Chamonix et Abondance ils le remplacent. Mais l'important est que dans les deux cas ils ont la connaissance des causes, non un simple avis consultatif. $\mathrm{Au} \mathrm{XV}^{\mathrm{e}}$ siècle d'ailleurs, la similitude n'échappait pas aux habitants d'Abondance et de Chamonix. En 1430, les premiers représentent à Amédée VIII que leur vallée est régie jure consuetudinario et non scripto, et qu'ils réclament seulement qu'on en use avec eux comme in ceteris patriis et locis territorii ducatus Sabaudie se regentibus consuetudine ${ }^{100}$. En revanche, lorsque nous voyons les Chamoniards prétendre en 1446 qu'ils ont les mêmes privilèges judiciaires que les probi homines plurium locorum, etiam ut Martignaci, Sancti Mauricii et aliorum plurium locorum ${ }^{101}$, cela ne contribue guère à éclairer notre lanterne. Car si la châtellenie de Martigny se trouve être la seule enclave coutumière à l'intérieur du Chablais savoyard, les terres de l'abbaye de Saint-Maurice d'Agaune sont clairement régies par le droit écrit ${ }^{102}$.

Surtout, il n'est pas si sûr qu'Abondance et Chamonix puissent sans plus d'examen être rangées dans les juridictions de droit coutumier. Il est vrai que les rapports entre seigneurs et paysans y sont régis par la coutume, laquelle est restée, comme nous le savons, largement orale jusqu'à la fin du Moyen Âge. Mais nos deux mandements se trouvent en Faucigny et en Chablais, c'est-à-dire qu'ils appartiennent clairement à la zone du droit écrit. Le droit civil, du reste, y manifeste à la fin du Moyen Âge l'influence $\mathrm{du}$ droit romain, notamment par l'usage du testament et des clauses de renonciations ${ }^{103}$. Quant à la procédure criminelle, si l'on excepte les garanties coutumières qu'on a évoquées, elle suit aussi le droit savant, singulièrement les Statuts publiés par les princes savoyards, qui témoignent du retour du droit romain depuis le XIII ${ }^{\mathrm{e}}$ siècle ${ }^{104}$.

C'est pourquoi les divergences ne sont pas moins nombreuses que les points de contact entre nos hautes vallées et les pays romands. Dans ces derniers en effet, les probi homines sont un petit groupes d'hommes convoqués par le juge ou le châtelain en tant qu'experts (periti) en coutume. Au XIV siècle, on tente de les choisir représentatifs de la société locale; mais on observe de plus en plus, à mesure qu'on avance dans le $\mathrm{XV}^{\mathrm{e}}$ siècle, une tendance à la professionnalisation de la fonction. Certains vont de cours en cours, donnent des consultations, offrent leurs services comme avocats. Ce sont souvent d'anciens officiers de justice, qui trouvent ainsi le moyen de rentabiliser l'expérience qu'ils ont acquise dans les cours seigneuriales. Tandis qu'à Chamonix ou à Abondance, nous avons vu que les termes de consuetudinarii ou de probi homines ne désignent pas un petit groupe de spécialistes, mais les communautés prises dans leur ensemble. Bien loin d'être convoqués à titre de jurisperiti, ils sont contraints de s'adjoindre les services de connaisseurs de la coutume ${ }^{105}$, mais surtout du droit savant. Dans le jugement précédemment évoqué du chamoniard Jean Pecluz, c'est clairement au droit écrit (jus commune) que fait référence le conseil juridique ${ }^{106}$. Tout en n'étant théoriquement pas liés par le conseil reçu, les prud'hommes de Chamonix et d'Abondance manifestent ainsi leur évidente incompétence.

Par ailleurs, les prud'hommes romands connaissent aussi bien les causes civiles que les causes pénales et, parmi ces dernières, celles qui regardent la haute, la moyenne et la 
basse justice. Tandis que les prérogatives des Chamoniards et des Abondanciers telles que nous venons de les décrire ne concernent que la juridiction de haute justice. Encore le juge de l'abbé d'Abondance est-il assisté de prud'hommes lorsqu'il fixe le montant des banna. Mais ils ne sont alors qu'en position d'assesseurs et l'on ne peut dire si leur avis n'est pas purement consultatif ${ }^{107}$. Quant aux Chamoniards, la connaissance des causes de moyenne et de basse justice et des causes civiles leur échappe complètement. Or les unes comme les autres forment l'immense majorité des procès dont nous avons la trace, et les prud'hommes y auraient beaucoup plus de compétences que dans les causes criminelles, la matière de ces affaires étant très souvent liée à la vie rurale. En toutes régions d'ailleurs, ne sont-ce pas ces causes que les seigneurs délèguent le plus volontiers ${ }^{108}$ ? Dans le mandement de Chamonix, elles sont du ressort du juge ordinaire ${ }^{109}$. Bien que mal éclairée par la documentation, la situation des habitants d'Abondance est peut-être assez proche à cet égard de celle des paysans romands. Les Chamoniards, eux, sont indubitablement au régime commun des communautés régies par le droit écrit.

70 Lorsqu'on les rapporte au contexte alpin, on mesure plus précisément l'originalité des prérogatives judiciaires des communautés de Chamonix et d'Abondance. Du point de vue de l'association au pouvoir normatif du seigneur, elles paraissent moins bien loties que nombre de communautés rurales des vallées voisines. Certes, les garanties procédurales dont elles disposent sont solides, mais assez communément partagées. Quant à leur participation à l'exercice de la justice, elle n'est pas sans faire songer aux pratiques judiciaires des pays romands de droit coutumier, alors que nos hautes vallées sont régies par le droit écrit, dans lequel leurs prud'hommes brillent par leur incompétence. Par ailleurs, l'exercice de la seule haute justice à l'exclusion de toutes les autres causes, ainsi que le fait que la connaissance des causes criminelle revienne aux communautés prises dans leur ensemble, sont des caractères proprement originaux.

Conclusion : des privilèges judiciaires au paradigme des libertés montagnardes

71 Les prérogatives judiciaires de nos deux communautés ainsi remises à leur juste place, le plus difficile reste à faire : formuler une hypothèse sur leur origine. Les Chamoniards $\mathrm{du} \mathrm{XV}^{\mathrm{e}}$ siècle les faisaient remonter, comme il est commun, à des temps immémoriaux ${ }^{110}$. Les historiens du XIX siècle leur ont emboîté le pas, y voyant une survivance des "libertés germaines». Dans les pays romands d'ailleurs, les probi homines attestés depuis la fin du XIII ${ }^{\mathrm{e}}$ siècle ne sont pas sans faire songer aux boni homines du haut Moyen Âge, dont le cartulaire de Lausanne fournit de rares exemples aux IX $\mathrm{IX}^{\mathrm{e}} \mathrm{X}^{\mathrm{e}}$ siècles et qui sont, nous assure-t-on, les « héritiers du tribunal des hommes libres de l'époque franque $»^{111}$. Reste qu'entre eux et les plus anciens prud'hommes attestés, il y a tout de même un vide documentaire de quatre siècles. À Chamonix et Abondance, la juridiction des prud'hommes est attestée à peu près au même moment que dans les pays romands. Mais le contexte antérieur n'y est pas le même que sur les rives du Léman.

72 À la fin du XI ${ }^{e}$ siècle, le comte de Genève affirmait que la vallée de Chamonix relevait de son comitatus, et qu'en la donnant à l'abbaye de La Cluse, il concédait également aux religieux omnia placita et banna. Cette dernière mention pourrait résulter d'une interpolation, car la plus ancienne copie qu'on possède de cet acte n'a pas été réalisée avant 1202. Le cas échéant, elle serait destinée à nier les droits des sires de Nangy, vassaux du comte de Genève, dont un représentant est déjà témoin de la donation originale. Les prieurs semblent avoir eu un certain mal à secouer la tutelle de cette 
famille seigneuriale, jusqu'à disparition de son dernier représentant au commencement du XIII ${ }^{e}$ siècle $^{112}$. Après la mort de celui-ci, les prieurs se sont fait reconnaître à plusieurs reprises par les comtes de Genève et les sires de Faucigny l'exercice de la haute et de la basse justice sur la vallée de Chamonix ${ }^{113}$. En tout état de cause, avant comme après qu'elle passe aux mains des bénédictins, la vallée a été soumise au pouvoir banal d'un ou plusieurs seigneurs.

Il en va de même à Abondance. En 1108, l'acte de constitution du temporel de l'abbaye réserve à la fois l'avouerie des sires de Féternes et le consentement du comte de Savoie, leur seigneur ${ }^{114}$. Dans une autre charte, probablement contemporaine, Guy de Féternes réserve encore son avouerie, tout en exemptant de son bannum le personnel (mercenarii) de l'abbaye, ce qui suppose que son ban s'exerçait normalement sur la vallée ${ }^{115}$. La lignée des Féternes s'éteint avant 1203. Les abbés d'Abondance semblent avoir alors réussi à éviter que l'avouerie passe entre les mains des comtes de Savoie, qui installent pourtant un châtelain à Féternes. Selon toute probabilité, c'est donc au XIII ${ }^{\mathrm{e}}$ siècle qu'ils ont obtenu l'exercice de la basse et de la haute justice sur la vallée ${ }^{116}$.

À Chamonix comme à Abondance, aussi haut que les textes permettent de remonter, c'est-à-dire au tournant des $\mathrm{XI}^{\mathrm{e}}$-XII ${ }^{\mathrm{e}}$ siècles, la seigneurie est donc en place. Celle des moines ne fait que succéder à celles des princes territoriaux et de leurs vassaux. La domination de ces derniers peut avoir été assez lâche, mais l'occupation du sol ne l'était pas moins. Que depuis des siècles nos hautes vallées aient été traversées, que leurs alpages aient été partiellement pâturés, cela ne fait aucun doute. Mais il ne s'ensuit pas qu'elles aient été occupées par des communautés paysannes structurées. Il faut renoncer au fantasme de libres communautés montagnardes qui n'auraient subi que tardivement le poids de la seigneurie. Ce sont les religieux qui, après leur propre installation, on fait appel à la colonisation paysanne en accord avec les seigneurs laïcs, ont établi dans les hautes vallées un peuplement dense et permanent, ont supervisé l'organisation du terroir. Tout ceci dans le cadre seigneurial ${ }^{117}$. Les prérogatives judiciaires qu'on constate à la fin du Moyen Âge ne sont donc certainement pas des vestiges de libertés antérieures à l'établissement de la seigneurie.

75 C'est pourquoi il ne faut pas s'étonner de rencontrer des textes qui, à peu d'années de distance, affirment aussi bien les droits de juridiction des communautés que ceux de leur seigneur; car les uns et les autres ne sont pas contradictoires. La sentence arbitrale de 1330 est, avons-nous dit, le premier témoin de l'exercice de la haute justice par les prud'hommes de Chamonix. Cela n'empêche pas le prieur d'affirmer en 1366 qu'il détient dans la vallée districtum de gentibus et delinquentibus, omniumque causarum et casuum civilium et criminalium inquisicionem, cognicionem et punicionem ${ }^{118}$. C'est que la justice y est toujours rendue en son nom. Simplement, il n'est pas libre de choisir son juge en matière criminelle, les prud'hommes étant alors de droit ses judices et cognitores. Leur procureur, lui, sera « juge du prieur élu par la communauté ${ }^{119}$. Celle-ci rend donc la justice au nom et au profit du prieur, de même que le juge ordinaire du monastère dans les causes qui sont de sa compétence. D’ailleurs, lorsque le cas se présente, ce dernier se démet de sa charge en leur faveur, par-devant notaire et pour une journée seulement. Au reste, cela lui permet souvent de jouer le rôle de conseiller juridique auprès des prud'hommes ${ }^{120}$.

76 Les syndics chamoniards ont soufflé le chaud et le froid, au gré des circonstances. En 1368 , nous les voyons dénier au prieur le droit de faire édifier des fourches patibulaires, et la sentence arbitrale rendue cette année-là abonde dans leur sens ${ }^{121}$. Elle reconnaît 
donc que les prieurs ne sont pas libres de leurs décisions en matière criminelle. D'ailleurs, en 1414, une fois son enquête faite, le prieur Antoine de Saint-Amour renonce à faire édifier les fourches de la discorde ${ }^{122}$. Mais en 1479 , face au vicechâtelain de Sallanches qui prétend enquêter sur les méfaits commis par des Valaisans dans le mandement de Chamonix, les syndics supplient le prieur d'évoquer à lui la connaissance de ces faits, en tant qu'il a toute juridiction sur la vallée ${ }^{123}$. C'est bien reconnaître qu'ils ne tiennent leur pouvoir judiciaire que de lui.

Que les prud'hommes de Chamonix jugent au nom du prieur; que ceux d'Abondance connaissent seuls les causes criminelles mais qu'ils soient seulement les assesseurs $d u$ juge de l'abbé dans les autres procès ; qu'enfin l'existence de communautés paysannes organisées avant l'arrivée des moines soit rien moins que certaine, tout cela laisse à penser que le tribunal des prud'hommes est dans nos deux vallées une émanation du tribunal seigneurial. Si l'on excepte le fait qu'ils en sont venus à évincer complètement le juge seigneurial dans les procès criminels, les coutumiers d'Abondance et de Chamonix ont finalement des prérogatives moins larges que ceux des pays romands. Et la règle qui impose l'unanimité des communautés ne vient jamais que d'un archaïsme qu'on constate dans tous les domaines où elles ont des décisions à prendre : jusqu'à la fin du Moyen Âge elles n'ont pas vraiment de personnalité juridique indépendante des membres qui les composent.

Compatibles en droit, les prérogatives judiciaires des monastères et des communautés ne l'étaient en fait que tant que leurs relations restaient correctes. Lorsqu'au $\mathrm{XV}^{\mathrm{e}}$ siècle elles se sont dégradées, la justice est devenue une pomme de discorde qui s'est ajoutée à d'autres, dont l'enjeu économique était autrement plus important. La transaction chamoniarde de 1493 réussit à rétablir la paix en empruntant au vocabulaire de la seigneurie foncière les formules qui définissent les droits de chaque partie : comme sur les alpages communaux, la communauté de Chamonix détient sur la justice criminelle le domaine utile, le prieur en conservant le domaine direct ${ }^{124}$.

Quant à la présence d'une procédure influencée par le droit coutumier dans une région où le droit écrit prédomine, c'est un état de fait que la documentation permet de constater, non d'expliquer, faute de remonter au-delà du XIVe siècle. Un chose est certaine: si l'on veut absolument faire intervenir la géographie, ce n'est pas le caractère montagneux des mandements de Chamonix et d'Abondance qu'il faut invoquer, mais le fait qu'ils se trouvent à la pointe septentrionale des pays de droit écrit et que l'influence des pays coutumiers a pu s'y exercer, contrariant les progrès du droit romain qui, selon Pierre Duparc, ne pénètre en Savoie que dans la première moitié du XIII ${ }^{\mathrm{e}}$ siècle ${ }^{125}$.

Les Alpes médiévales, en tout cas, ne sont pas un espace de liberté au centre d'une Europe «féodale », moins encore une zone de non-droit. L'air de la montagne ne rend pas libre, non plus qu'il ne porte à l'égalité, n'en déplaise à Jean-Jacques Rousseau. Qu'on relise le célèbre Pacte de $1291^{126}$, qui donnait naissance à la plus riche d'avenir des "confédérations de vallées» alpines, puisqu'elle était grosse de la nation suisse. Que propose-t-il comme remède à la malice des temps? Que les communautés montagnardes des vallées de Schwyz, Uri et Unterwald se portent assistance, certes, mais principalement pour y réprimer les criminels ; que chacun vive selon sa condition, obéisse à son juge, respecte sa foi envers son seigneur et acquitte les services qu'il lui doit. Rien d'autre, donc, que le respect de l'ordre seigneurial. Ordre auquel les montagnes médiévales ont été soumises à l'égal du plat pays. Ordre qui tolérait les 
privilèges judiciaires finalement limités des communautés de Chamonix et d'Abondance, alors que l'État moderne allait les juger incompatibles avec l'ordre nouveau qu'il instaurait. C'est ainsi que le XVI ${ }^{e}$ siècle les vit disparaître.

Carte de localisation des principales communautés citées

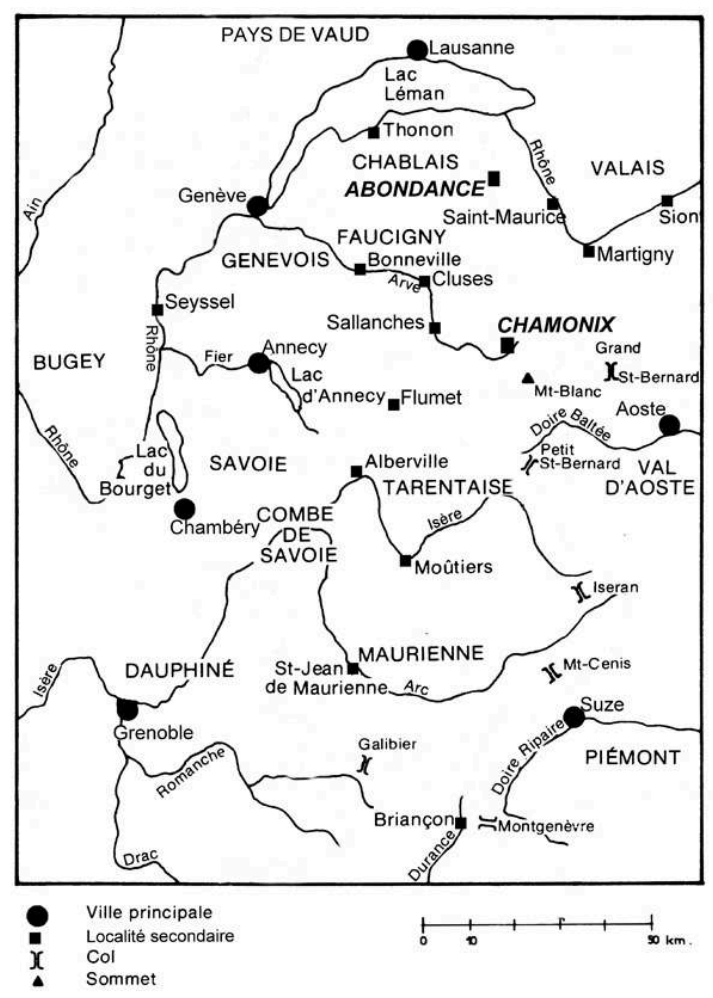

\section{NOTES}

1.P. Vaillant, « Les origines d'une libre confédération de vallées : les habitants des communautés briançonnaises au XIII siècle ", Bibliothèque de l'École des Chartes, 125, 1968, pp. 301-348. J.-F. Bergier, Guillaume Tell, Paris, 1988. J.-P. Boyer, Hommes et communautés du haut pays niçois : La Vésubie (XIII ${ }^{e}-X V^{e}$ siècle), Nice, 1990. L'histoire médiévale de l'Andorre vient d'être renouvelée par R. Viader, $L^{\prime}$ Andorre du IX ${ }^{e}$ au XIV siècle, Toulouse, 2003.

2.C'est notamment la thèse de G. Pérouse, « Les communes et les institutions de l'ancienne Savoie, d'après les archives communales ", Inventaire sommaire des archives départementales de la Savoie antérieures à 1793, série E, archives communales, Chambéry, 1911, pp. I-C.

3.A. Perrin, Histoire de la vallée et du prieuré de Chamonix du $X^{\mathrm{e}}$ au XVIII siècle, Chambéry, 1887. 
4.A. Perrin, « La justice criminelle dans les hautes vallées des Alpes au Moyen Âge », Congrès des Sociétés savantes de Savoie, 1879, pp. 77-86.

5.Ainsi le chanoine Mercier voyait-il sur le visage des Abondanciers « cet air d'indépendance et de fierté qui caractérise l'homme libre ». Il ne s'en étonnait pas, car en éditant (correctement d'ailleurs, même s'il n'a eu souvent à sa disposition que des copies du XVIII siècle) les textes contenant les franchises de la vallée, il avait appris « que ce sont là les descendants d'une race d'hommes qui pendant les siècles de la féodalité ont su conserver leur indépendance et se gouverner par eux-mêmes, exercer les attributions de la justice, et faire de leur vallée une oasis pour la liberté dans ce Moyen Âge qu'on appelle les siècles de la servitude » (J. Mercier, L'abbaye et la vallée d'Abondance, Annecy, 1885 (Mémoires et documents de l'Académie salésienne, 8), p. 2). 6.H. Falque-Vert, Les hommes et la montagne en Dauphiné au XIII siècle, Grenoble, 1997, pp. 233-246, 257-264.

7.N. Carrier, La vie montagnarde en Faucigny à la fin du Moyen Âge. Économie et société, fin XIII ${ }^{e}$-début XVI siècle, Paris, 2001, pp. 379-431.

8.Infra, n. 18, 40, 43, 48.

9. En effet, il arrive souvent dans les Alpes que les mêmes familles de franchises s'applique aussi bien à des localités urbaines que rurales. Pour ne prendre qu'un exemple, la charte de la ville d'Aoste est le modèle de bien des libertés rurales valdôtaines (E.-E. Gerbore, « Les plus anciennes franchises rurales valdôtaines (1270-1311) : types et problèmes ", Liberté et libertés. VIII centenaire de la charte de franchises d'Aoste. Actes du colloque international d'Aoste, 20 et 21 septembre 1991, éd. A. Fosson et J.-G. Rivolin, Aoste, 1993, pp. 115-131.)

10.J.-Y. Mariotte, « Les origines du prieuré de Chamonix 》, Bibliothèque de l'École des Chartes, 136, 1978, pp. 241-269.

11.Aujourd'hui communes de Chamonix, Vallorcine et Servoz, c. Chamonix, arr. Bonneville, Haute-Savoie.

12.Sur les limites du temporel initial du monastère, J.-Y. Mariotte, « Note sur la seigneurie monastique en Savoie. Le problème de l'avouerie ", Mémoires de la société pour l'histoire du droit et des institutions des anciens pays bourguignons, comtois et romans, 1977, pp. 87-95, cf. p. 93 n. 1.

13.En 1359, le comte Amédée VI exempte l'abbaye et ses dépendants d'un droit de garde qu'ils devaient payer annuellement entre les mains du châtelain comtal d'Evian et Féternes. Il est alors question des homines parrochiarum superioris et inferioris vallis Habundantie (L.-E. Piccard, L'abbaye d'Abondance et la vallée du même nom, t. 2, Documents, Thonon, 1905 (Mémoires et documents de l'Académie du Chablais, t. 19), doc. 5). La paroisse supérieure est manifestement celle de La Chapelle des Frasses, aujourd'hui commune et paroisse de la Chapelle d'Abondance, c. Abondance, arr. Thonon-les-Bains, Haute-Savoie, dont la paroisse de Châtel s'est détachée au commencement du XVIII ${ }^{e}$ siècle, act. Com. et c. Châtel, arr. Thonon-les-Bains, Haute-Savoie (J.-Y. Mariotte, Histoire des communes savoyardes. Haute-Savoie, t. 1, Le Chablais, Roanne, 1980, p. 205). La paroisse inférieure ne peut être que celle d'Abondance.

14.L. Charvet, Recherches sur l'abbaye d'Abondance, Lyon, 1863, pp. 52-61. J. Mercier, op. cit., pp. 74-91.

15.Sur la communauté de Chamonix, mise au point récente dans N. Carrier, La vie montagnarde, op. cit., pp. 422-424, 506-518. 
16.J.-A. Bonnefoy, A. Perrin (éd.), Documents relatifs au prieuré et à la vallée de Chamonix, Chambéry, 2 vol., 1879-1883 (Académie de Savoie, Documents, t. 3 et 4), t. 1, doc. 80. Les textes tirés de ce recueil seront dorénavant cités sous la forme Bonnefoy, doc. 80 .

17. Bonnefoy, doc. 110.

18.Le débat portait sur la formule suivante : Quod probi homines dicte vallis, malefactores ad penam corporalem et non civiles delinquentes cum consilio dictis probis ministraturo per ipsum dominum priorem quando casus evenit, sumptibus ipsius domini prioris, judicare debent insequendo ipsum consilium et non alias. Les procureurs de la communauté protestent quod hoc fuit error scriptoris, propter quod requirunt ipsam clausulam corrigi et emendari, quoniam ipsi homines, secundum consuetudinem et capitula ipsorum, judicare debent malefactores judicandos in dicta valle ad penam corporalem, presente consilio per dictum dominum priorem sibi ministraturo suis sumptibus, sed non quod sint astricti ad insequendum dictum consilium, sicut in ipsa clausula continetur, ut asserunt (Bonnefoy, doc. 119).

19.Bonnefoy, doc. 151 (1458, sorcellerie), 148 (1459, idem), 161 (1462, idem), 175-177 (1470, adultère, inceste, agression contre le curé de Vallorcine et voies de fait contre les officiers du prieur).

20.Et non Guillaume II de la Ravoire, contrairement à ce que nous avons écrit dans notre Vie montagnarde, op. cit., p. 512. Guillaume II est le second fils de Guillaume $\mathrm{I}^{\mathrm{er}}$. Dernier prieur de Chamonix, il succède à son frère Jacques de 1493 à 1519 (v. infra).

21.N. Carrier, La vie montagnarde, op. cit., p. 517.

22.Bonnefoy, doc. 196.

23.Bonnefoy, doc. 197b (1494).

24.Bonnefoy, doc. 194a, b, c : Jacques Métral, d'Arbignion en Valais, cherche à se débarrasser de son frère Collet, avec lequel il est décidé à ne pas partager l'héritage paternel. Sa femme Jeannette, sur le conseil de siens parents originaires de Chamonix, prend contact avec deux Chamoniards résidant à ce moment à Martigny, Pierre Veytet et Jacques Bontemps, qui acceptent et exécutent le contrat contre la somme de 19 florins. Jacques Bontemps portait alors calligas rubeas cissas sub genu et bonetum rubeum a latere. La couleur rouge était le signe de ralliement des révoltés faucignerans de 1492, auxquels la chronique a donné le nom de « Robes rouges » (N. Carrier, La vie montagnarde, op. cit., pp. 523-530).

25.Bonnefoy, doc. 198.

26.Bonnefoy, doc. 199.

27.Bonnefoy, doc. 206-207.

28.Au Moyen Âge, le terroir de la paroisse de Vacheresse correspondait à celui des deux communes actuelles de Vacheresse et Bonnevaux, c. Abondance, arr. Thonon-les-Bains, Haute-Savoie. La paroisse de Bonnevaux s'est détachée de celle de Vacheresse au XVIII $^{\mathrm{e}}$ siècle (J.-Y. Mariotte, Histoire des communes, op. cit., pp. 202). Sur l'acquisition des droits seigneuriaux par les abbés d'Abondance à Vacheresse, supra n. 14 .

29.J. Mercier, " Pièces justificatives et documents », op. cit., doc. 5. Les textes tirés de ce recueil seront dorénavant cités sous la forme Mercier, doc. 5 .

30. Cet acte est également mentionné dans un inventaire des titres de l'abbaye rédigé en 1687 (L. Charvet, op. cit., p. 80, cf. p. 53).

31.Une sentence arbitrale rendue par Amédée VIII en 1430 (v. infra), évoque aussi une transaction dans laquelle les habitants de la communauté se seraient engagés à ne pas se réunir hors de la présence d'un représentant de l'abbé (Mercier, doc. 12). Il est impossible de rien savoir de la date de ce texte, sinon qu'il est antérieur à 1430 .

32.J. Mercier, op. cit., pp. 141-142. 
33.Infra, n. 60.

34.Mercier, doc. 6 .

35.Mercier, doc. 11. L'arbitrage de 1419 et la transaction de 1420 sont connus par l'inventaire de 1687 cité ci-dessus (L. Charvet, op. cit., p. 65).

36.Sur l'auciège, cette redevance en fromage due pour la concession des chalets d'alpage, cf. P. Duparc, « Une redevance féodale alpestre : l'auciège », Bibliothèque de l'École des Chartes, 105, 1944, pp. 99-122, à actualiser par N. Carrier, La vie montagnarde, op. cit., pp. 339-343.

37.Mercier, doc. 12.

38.Dans la Savoie de la fin du Moyen Âge, un métral est un « officier » (sergent) seigneurial chargé de diverses fonctions de police et de perception des redevances. 39. Quoad casum Mermeti Burnier qui, propter mortem dicti Berbillaux, mistralis Habundancie, per nonnullos dicte vallis homines interempti et occisi, captus fuerat per officiarios dicti domini abbatis et qui in carceribus existens, ut communiter fertur, morte sibi consciens laqueo se suspendisse dicitur ; quia dicti homines et potissime parentes et amici dicti Burnier, asserentes ipsum mortuum fuisse in dictis carceribus dolo, culpa seu diffectu dictorum officiariorum, petebant ipsum Burnier aut saltem ipsius corpus et cadaver sibi reddi et restitui et inde sibi de culpabilibus, si qui forent, mortis ipsius justiciam ministrari ; pro parte vero dicti Dni. abbatis, ejusque conventus et officiariorum [...] contrarium asserebatur, prosequebanturque dicti officiarii alios culpabiles et obnoxios de interemptu seu morte dicti Berbillaux ut via juridica punirentur (Sentence arbitrale du duc Amédée VIII, Mercier, doc. 13).

40.Cum vallis Habundancie regatur jure consuetudinario et non scripto et per tanta tempora cujus inicii non existat in contrarium memoria, quando et quociescumque occurrit sentencia ferenda in aliqua criminali corporali causa infra limites juridicionis vallis Habundancie, cognicio fit per consuetudinarios et probos homines vallis ; secundum quorum cognicionem proceditur, judicatur et fit exequucio, prout et in ceteris patriis et locis territorii ducatus Sabaudie se regentibus consuetudine. Contingit quod reverendus dominus abbas et ejus officiarii sine ulla consuetudinariorum cognicione suspendi fecit Mermetum Burnier, de ipsa valle, contra dictam consuetudinem et eam infrigendo (Sentence arbitrale du duc Amédée VIII, Mercier, doc. 12).

41.Mercier, doc. 12-13. Nous sommes à mi-chemin entre l'arbitrage amiable et la décision judiciaire : la sentence en effet prévoit une amende de mille livres au contrevenant, la moitié revenant au duc et l'autre moitié à la partie lésée.

42.Mercier, doc. 13.

43. Habita prius super hoc diligenti informatione, declaraverunt quod cogniciones causarum criminalium corporalium [...] fiant [...] per probos homines et consuetudinarios ipsius vallis, executiones vero dampnatorum [...] pertineant officiariis predictorum dominorum abbatis et religiosorum. [...] Cause tamen appellacionum et nullitatum devolvantur et devolvi debeant ad abbatem et conventum seu eorum judicem appellacionum, per quem tales appellaciones et nullitatum cause audiri cognosci, decidi et sine debito terminari debeant, possint et valeant (Mercier, doc. 12).

44.Mercier, doc. 7.

45. À notre connaissance, on ne possède plus aujourd'hui que des analyses de ce texte. La plus précise et, semble-t-il, la plus fiable, est celle de J. Mercier, op. cit., pp. 155-157. Il s'agit bien d'une transaction et non d'une sentence arbitrale.

46. Mercier, doc. 22 (1476, cf. infra n. 48), 23 (1522, cf. infra n. 49), 24 (acquittement d'une femme accusée de meurtre, 1557), 25 (condamnation à mort d'une sorcière, 1502). 
L.-E. Piccard, Histoire de Thonon et du Chablais, des origines à la Révolution française, Annecy, 1882 (Mémoires et documents de l'Académie salésienne, 5), doc. 10 (sentence de mort contre une femme, 1483 ; fustigation et bannissement d'un homme, 1562). 47.Bonnefoy, doc. 177 .

48. Comparuerunt coram [...] honestis viris Mermeto Maxi, Mermeto de Porti, Jaqueto Brelaz et Guilliermo Fabri, veluti procuratoribus et scindicis, et [suit une liste de 105 personnes], probis hominibus et consuetudinariis vallis Abundantie, quibus [pertinet] cognitio causarum criminalium corporalium secundum consuetudinem ipsius vallis, [...] providus vir Petrus Brelat, [...] velut procurator et procuratorio nomine, [...] administrator insigni monasteris B. Mariae Abundantiae [...] requirens [...] consuetudinarios de et super quodam processu criminali ex officio curie dictorum dominorum administratorum et religiosorum eorumque monasterii contra Johannem Marollaz, [...] cognosci, sententiari, jus dici et definiri ex una parte ; et dictus Johannes Marrolaz delatus, requirens equidem per ipsos consuetudinarios de et super ipso processu cognosci et se a contentis in eodem absolvi et liberari, ex alia parte. Quibus partibus auditis per ipsos consuetudinarios, lectis eis prius lingua romana per supranominatum procuratorem et expositis articulis dicti processus et responsionibus per ipsum delatum factis [...], participato prius etiam consilio cum peritis in jure et consuetudine, [...] idcirco, voce et organo dicti Mermeti Maxi et eo sic in vulgari sermone referente, in aliorum supranominatorum proborum hominum et consuetudinarium presentia, cognoverunt et sententiaverunt [...] ipsum Johannem delatum esse bannitum [...] de valle Abundantiae per unum annum (Mercier, doc. 22).

49.Cf. cet acquittement d'un homme accusé de l'assassinat du curé d'Abondance en 1522 : Viso processu tuo in curia Abondantiae [...] contra te formato, visis responsionibus tuis [...], nos igitur [suit la liste des consuétudinaires présents, en tête desquels les quatre syndics], pro tribunali in loco Passus sedentes, [...] participato prius peritorum consilio, voce et organo praedicti Johannis Exevuas [le premier nommé des syndics] et sic et vulgari sermone referente in aliorum proborum hominum presentia, cognoscimus unanimiter et sententiamus te [...] esse absolvendum et liberandum (Mercier, doc. 23).

50.Cum communitas proborum virorum vallis et tocius mandamenti et juridicionis Campimuniti [...] habeant [...] jus, usumque et consuetudinem [...] de et super delinquentibus et commictentibus casus criminales tanquam judices et cognitores domini prioris Campimuniti [...] sentenciandi, pronunciandi, judicandi, condempnandi et absolvendi, [...] facto prius, formato et completo processus per clericum curie dicti domini prioris et ejus prioratus aut alterum sufficientem potestatem ad hoc habendum... (Bonnefoy, doc. 158, 1459). Abondance : cf. supra, n. 40 et 43 .

51.Bonnefoy, doc. 177 (1470).

52.Bonnefoy, doc. 161 (1462).

53.C'est le cas dans le procès (très politique) de Michel Berthoud, condamné à mort en 1495 pour avoir tenté d'assassiner le prieur : il est probable que les syndics souhaitaient manifester clairement que la communauté se désolidarisait du criminel : Dictis syndicis necnon Johanneto Bruneti, parte predicte communitatis ad infrascripta electo sedentibus in bancha curie, [...] dictus Johannetus supra electus, in presentia etiam sepedictorum syndicorum, deque ipsorum voluntate et consensu [...] condamnavit et sententiavit eundem Michaelem [...] ad furchas [...] suspendendum. [...] Quibus premissis sic actis, prefati sindici cum dicto electo nomine dicte communitatis eumdem delatum remiserunt [...] vicecastellano supranominato pro executione fieri mandanta. Les syndics ont ici un rôle actif, conjointement avec le procureur-juge, dans la remise du condamné au châtelain (Bonnefoy, doc. 199). 54.Abondance : supra, n. 42 et 43. Chamonix : Omnis inquisitio fienda in et penes juridictionem [...] prioratus Campimuniti ac quorumcumque processuum formatio, 
criminosorumque captio, detentio, carceratio et examinatio usque ad sententiam diffinitivam ferendam exclusive sit et pleno jure pertineat ad dominum priorem (Bonnefoy, doc. 196). 55.Supra, n. 48.

56. Alter ex officiis domini prioris [...], lapsis [...] decem diebus, infra duos dies proxime sequentes teneatur et debeat notificare uni aut duobus ex sindicis dicte communitatis detentionem criminosi seu criminosorum ; qui sindicus vel sindici si voluerint [...] habeant eligere [...] tres ex probis hominibus dicte communitatis non supectos qui jurare habeant de non revellandis confessionibus et interrogationibus [...] factis ; qui probi homines si voluerint [...] interesse possint et debeant omnibus interrogationibus (Bonnefoy, doc. 196).

57.Bonnefoy, doc. 175-176, (1470).

58.Le cas savoyard est étudié par N. Carrier, « Une justice pour rétablir la " concorde » : la justice de composition dans la Savoie de la fin du Moyen Âge ", Le règlement des conflits au Moyen Âge. Actes du XXXI ${ }^{e}$ congrès de la SHMESP (Angers, 2000), Paris, 2001, pp. 237-257.

59.Dominus prior [...] tales carceratos [...] sine aliqua tortura [...] componere possit ad penam pecuniariam et etiam eis indulgere in casibus et delictis criminalibus in quibus potest et debet pena corporis vel sanguinis infligi, satisfacto prius per dictos criminosos parti lese de juribus et interesse suis (Bonnefoy, doc. 196).

60. Mistralis noster plura banna obscura male discussa ab ipsis hominibus exigit et extorquet, absque eo quod ipse velit cognitionem nostri causidici expectare, qui causidicus, participato proborum hominum consilio, tenetur, ut asserunt, ipsa banna declarare (Mercier, doc. 5). 61.Comme en témoignent les cahiers d'assises du prieuré de Chamonix qu'on a conservés, cf. infra, n. 109.

62.Bonnefoy, doc. 97 (composition pour un meurtre, 1383), 147-148 (composition pour un vol dans l'église et protestation des syndics, le prieur rétorquant que recevoir une composition n'est pas rendre une sentence, 1446), 179 (composition d'un homme pour divers délits et pour le suicide de son fils, 1473), 204 (composition pour meurtre, 1516). 63.Bonnefoy, doc. 86 (vol d'une jument, 1364), 99 (vol d'un cheval, 1388), 101 (vol de divers objets, 1396), 102 (vol d'or et d'argent, 1399), 112-113 (condamnation à mort pour assassinat, 1417), 128 (sentence de contumace contre un homme de Montjoie, 1431).

64.Si aliquando dicta communitas aliquos malefactores judicaverit et ad penam corporalem condempnaverit, scientibus [...] dominis prioribus [...] patientibusque et non contradicentibus, hoc fuit in paucis casibus nec potuit predicto prioratui prejudicare (Bonnefoy, doc. 196 (1493)).

65.Le terme bannum a donc trois sens : la pénalité préalablement prévue par le droit, l'infraction sanctionnée par cette pénalité, la pénalité réellement perçue, soit sous forme de composition, soit sous forme d'amende.

66.J.-F. Poudret, Coutumes et coutumiers. Histoire comparative des droits des pays romands du $X I I I^{e}$ à la fin du XVI $I^{e}$ siècle, Berne, 1998, t. 1, pp. 117-118, 133, 166-167.

67.P. Vaillant, Les libertés des communautés dauphinoises (des origines au 5 janvier 1355), Paris, 1951, p. 299.

68. « Deuxième centurie de documents historiques inédits publiés par Auguste Dufour et Charles Rabut : chartes municipales des pays soumis à la Maison de Savoie en deçà des Alpes ", Mémoires et documents de la Société savoisienne d'histoire et d'archéologie, 23, 1885 , doc. 12 , p. 245. 
69.J.-Y. Mariotte, " La charte de fondation de Flumet, source du droit de Fribourg ", Mémoires de la société pour l'histoire du droit et des institutions des anciens pays bourguignons, comtois et romans, 1970-1971, pp. 73-92, cf. p. 92. Il est vrai que cette charte est remplacée en 1307 par une autre beaucoup moins avantageuse de ce point de vue, qui ramène le bourg de Flumet au niveau des autres villes franches faucignerandes, où les bourgeois paraissent exclus du pouvoir normatif.

70.J.-M. Carbasse, Introduction historique au droit pénal, Paris, 1990, pp. 85-91.

71.60 sous genevois représentent quatre à six mois de salaire agricole sur la réserve des comtes de Genève au commencement du XIV ${ }^{\mathrm{e}}$ siècle (G. Détraz, « Les corvées dans le Genevois au XIV siècle », Revue Savoisienne, 1990, pp. 80-96, cf. p. 93).

72.J.-G. Rivolin, « Les franchises d'Aoste : la charte de Thomas I ${ }^{\text {er }}$ de Savoie », Liberté et libertés, op. cit., pp. 99-114, cf. pp. 113-114.

73.R. Mariotte-Löber, Ville et seigneurie. Les chartes de franchises des comtes de Savoie, Annecy, 1973, pp. 77-85.

74.P. Vaillant, Les libertés, op. cit., p. 293.

75.E.-E. Gerbore, art. cit., pp. 117-118.

76.J.-F. Poudret, « Le rôle des plaids généraux dans la formation, la transmission et l'enregistrement de la coutume d'après les sources romandes du Moyen Âge », Mémoires de la société pour l'histoire du droit et des institutions des anciens pays bourguignons, comtois et romans, 40,1983, pp. 177-193 et P. Dubuis, « La « préhistoire 》 des communautés rurales dans le Valais médiéval », Liberté et libertés, op. cit., pp. 1-8. 77. Original perdu, connu par une copie de 1438, Arch. de l'abbaye de Saint-Maurice d'Agaune, T 15/1/2.

78.Item, omnia statuta et ordinationes hactenus factas per communinates castellanie Tharentasie super communibus pascuis montium et planorum dicte castellanie si nobis et rei publice utile non fuerunt [...] revocamus (« Copie des franchises des habitants de la Tarentaise au-dessus du Saix, accordées par les princes de Savoie, en 1391 », Académie de la Val d'Isère. Documents, 1, 1866, pp. 399-410, cf. p. 408).

79.Bonnefoy, doc. 115,132 et 137.

80.Bonnefoy, doc. 190-191.

81.Bonnefoy, doc. 189 (1484).

82.J.-F. Poudret, Libertés et franchises dans les pays romands au Moyen Âge. Des libertés aux droits de l'homme, Lausanne, 1986 (Cahiers de la Renaissance vaudoise, 113), pp. 66-75 et « Le concept de liberté au Moyen Âge », Liberté et libertés, op. cit., pp. 27-38.

83.R. Mariotte-Löber, op. cit., p. 77. P. Vaillant, Les libertés, op. cit., pp. 293-296.

84.Bonnefoy, doc. 45, art. 11.

85.Bonnefoy, doc. 93, art. 2 et 4 .

86.Mercier, doc. 5 .

87.0fficiarii [...] abbatis dietim capiunt de probis hominibus vallis et atrocibus ac impiis modis eos pertractant et cruciatus afferunt in corporibus eorum, adeo quod metu minarum carceris et tantorum cruciatuum compelluntur concordare ad magnas pecuniarum summas (Mercier, doc. 12).

88.Ibid.

89.J. Mercier, op. cit., p. 157.

90.J.-F. Poudret, « Le concept de liberté », art. cit., p. 29. 
91.Jusqu'en 1475, ce bailliage a regroupé les territoires correspondant à l'actuel Chablais haut-savoyard et au Valais occidental. Il était régi par le droit écrit, à l'exception de la seule châtellenie de Martigny (J.-F. Poudret, Coutume, op. cit., t. 1 p. 29). 92.J.-F. Poudret, Coutume, op. cit., t. 1, pp. 1-82. P. Duparc, « La pénétration du droit romain en Savoie (première moitié du XIII ${ }^{\mathrm{e}}$ siècle) », Revue historique du droit français et étranger, 1965, pp. 22-86.

93.P. Vaillant, « Les franchises », art. cit., p. 395. Cf. L. Falletti, « Éléments d'un tableau chronologique des franchises de Savoie », Revue savoisienne, 78, 1937, p. 142.

94.Si quis autem infra predictum terminum alicui vim intulerit vel vulneravit vel aliquo modo percusserit, dannum et injuriam leso restituat et ad cognitionem nuncii comitis et habitatorum [domino] sastifaciat (sic) (J.-G. Rivolin, art. cit., p. 113).

95.J.-Y. Mariotte, « La charte de fondation de Flumet », art. cit., pp. 84, 86. Cette charte déroge au droit de la région car elle est influencée par le droit de Fribourg.

96. A. Dufour, F. Rabut, Histoire de la commune de Flumet suivie de documents inédits relatifs à cette localité, Chambéry, 1867 (Mémoires et documents publiés par la Société savoisienne d'histoire et d'archéologie, 11), doc. 2, pp. 107-116. Par la suite, les bans conservés par centaines dans les comptes de la châtellenie de Flumet ne portent aucune trace de l'intervention des bourgeois du lieu dans leur fixation. (Nous remercions Nathalie Favre-Bonvin de nous avoir fourni ce renseignement.)

97.R. Mariotte-Löber, op. cit., n. 3, p. 80.

98.P. Vaillant, Les libertés, op. cit., pp. 299-305.

99.Sur ce qui suit, cf. J.-F. Poudret, Coutume, op. cit., t. 1, pp. 259-306, où l'on trouvera citée la bibliographie antérieure.

100.Supra, n. 40.

101.Bonnefoy, doc. 148.

102.J.-F. Poudret, Coutume, op. cit., t. 1, pp. 77-79.

103. Comme en témoignent par exemple les minutiers chamoniards conservés pour le $\mathrm{XV}^{\mathrm{e}}$ siècle (Arch. dép. Haute-Savoie, 2E 3012-3016).

104.L. Chevailler, Recherches sur la réception du droit romain en Savoie des origines à 1789, Annecy, 1953, pp. 21-112.

105.Supra, n. 48.

106.Supra, n. 47.

107.Supra, n. 60.

108.M. Bourin, R. Durand, Vivre au village au Moyen Âge. Les solidarités paysannes du XI ${ }^{e}$ au XIII ${ }^{e}$ siècle, Paris, 1984, pp. 182-191.

109.Plusieurs cahiers d'assises ont été conservés pour le mandement de Chamonix. Ils enregistrent les banna concordata ou condemnata levés par le juge au profit du prieur (Arch. dép. Haute-Savoie, 10 G 223 (2 juin 1467, 26 janvier 1471, 5 novembre 1476), 10 G 224 (11 février 1476)).

110.Cum communitas dicti loci Campimuniti et homines ejusdem habeant et habuerint per tempora hominum memoriam excedentia [...] jus et potestatem condemnandi malefactores puniandos pena corporali... (Bonnefoy, doc. 199 (1495)).

111.C. Lauranson-Rosaz, « La romanité du Midi de l'an Mil. Le point sur les sociétés méridionales », La France de l'an Mil, dir. R. Delort, Paris, 1990, p. 57.

112.J.-Y. Mariotte, « Les origines », art. cit.

113.Bonnefoy, doc. 34 (1289), 44 (1291), 73 (1319).

114.J.-Y. Mariotte, « Note sur la seigneurie monastique », art. cit., pp. 89 et 94. 
115.Ibid., p. 89. Edition fautive et datation erronée de ce texte dans L.-E. Piccard, L'abbaye d'Abondance, op. cit., t. 2, doc. 1.

116. ''est une chose acquise en tout cas en 1325 (Mercier, doc. 5). Cela ne s'est peut-être pas fait sans mal, si l'on en juge par les difficultés que les abbés ont rencontresé pour faire admettre leur juridiction de haute-justice sur le village de Saint-Gingolph, riverain du Léman, où ils ont eu à batailler contre les châtelains d'Evian-Féternes, de Chillon et des Allinges entre 1203 et 1322 (Mercier, doc. 4).

117.Le cas de Chamonix et du haut Faucigny est étudié dans N. Carrier, La vie montagnarde, op. cit., pp. 173-183. Nous développons ce thème dans « Les moines et la montagne dans les Alpes nord-occidentales au Moyen Âge », Montagnes médiévale. Actes du XXXIVe congrès de la SHMESP (Chambéry, 2003), Paris, à paraître.

118.Bonnefoy, doc. 89 .

119.Ainsi Jacques Bollet, qui envoie au supplice plusieurs sorciers au bûcher en 1462 , est-il « judex [...] domini prioris per [...] communitatem electus, nomine et de voluntate predicte totius communitatis, vel saltim majoris partis ibidem existentis » (Bonnefoy, doc. 161). 120.Bonnefoy, doc. 153 (1458) et 157 (1459). Cela n'est pas contraire à la coutume qui spécifie que le juge doit être choisi extra terram. Car le juge du prieuré est un gradué en droit qui se loue au prieur de Chamonix aussi bien qu'à d'autres seigneurs et qui ne vient donc que périodiquement dans le mandement.

121.Bonnefoy, doc. 93.

122. Bonnefoy, doc. 110 .

123. Bonnefoy, doc. 183.

124.Item transegerunt jam dicte partes [...] quod dicti communitas et probi homines [...] teneant predictas absolutionem et condemnationem ipsisque utantur de directo prefatorum domini prioris et ejus prioratus dominio (Bonnefoy, doc. 196).

125.P. Duparc, « La pénétration », art. cit.

126. Cité dans J.-F. Bergier, Guillaume Tell, op. cit., pp. 336-338.

\section{AUTEUR}

\section{NICOLAS CARRIER}

Centre d'histoire médiévale, Université Lyon-III 